\title{
Overcoming duality: the fused bousfieldian function for modeling word production in verbal fluency tasks
}

\author{
Felicitas Ehlen $^{1}$ - Ortwin Fromm ${ }^{1}$ - Isabelle Vonberg ${ }^{1}$ - Fabian Klostermann ${ }^{1,2}$
}

Published online: 29 December 2015

(C) Psychonomic Society, Inc. 2015

\begin{abstract}
Word production is generally assumed to occur as a function of a broadly interconnected language system. In terms of verbal fluency tasks, word production dynamics can be assessed by analyzing respective time courses via curve fitting. Here, a new generalized fitting function is presented by merging the two dichotomous classical Bousfieldian functions into one overarching power function with an adjustable shape parameter. When applied to empirical data from verbal fluency tasks, the error of approximation was significantly reduced while also fulfilling the Bayesian information criterion, suggesting a superior overall application value. Moreover, the approach identified a previously unknown logarithmic time course, providing further evidence of an underlying lexical network structure. In view of theories on lexical access, the corresponding modeling differentiates task-immanent lexical suppression from automatic lexical coactivation. In conclusion, our approach indicates that process dynamics result from an increasing cognitive effort to suppress automatic network functions.
\end{abstract}

Keywords Math modeling and model evaluation · Word production · Verbal fluency

Since the landmark works of 19th-century scientists like Broca, Wernicke (1874), and Lichtheim (1885) on speech

Felicitas Ehlen and Ortwin Fromm contributed equally to this work.

Felicitas Ehlen

felicitas.ehlen@charite.de

1 Department of Neurology, Motor, and Cognition Group, Charite University Medicine Berlin, Campus Benjamin Franklin, Berlin, Germany

2 Berlin School of Mind and Brain, Berlin, Germany and language processing, both the linguistic and neuroscientific view on language function has undergone many refinements. Most prominently, the language system's network structure that had already been suggested implicitly by these early approaches has been further elucidated. In so doing, the functions and spatiotemporal characteristics of corresponding anatomical structures have been thoroughly described by means of complementary techniques, including clinical approaches, neuroimaging, and electrophysiological assessments (e.g. Braun et al., 2015; Costa, Strijkers, Martin, \& Thierry, 2009; Indefrey \& Levelt, 2000; Jackson, Hoffman, Pobric, \& Lambon Ralph, 2015; McDermott, Petersen, Watson, \& Ojemann, 2003; Saur et al., 2008; Schuhmann, Schiller, Goebel, \& Sack, 2009; Schwartz, Dell, Martin, Gahl, \& Sobel, 2006; Wahl et al., 2008; Wilson, Isenberg, \& Hickok, 2009). Altogether, they depict a diversely spread out language system (Binder, Desai, Graves, \& Conant, 2009; Indefrey, 2011; Indefrey \& Levelt, 2004; Pulvermuller, 1999). Complex interactions between the semantic, sensory, and motor speech systems have been hypothesized for both language perception and production (Hickok, 2012; Hickok \& Poeppel, 2007; Walker \& Hickok, 2015; cf. Saur et al., 2008). One important step in the process of language production is the correct selection and production of each word. In this regard, classical psycholinguistic models have argued that word production occurs on separate yet interconnected processing levels (either in a serial, cascade, or parallel fashion), encompassing the "conceptualization," "formulation," and "articulation" of respective lexical items (e.g. Caramazza, 1997; Dell, 1986; Levelt, 1999; Levelt, Roelofs, \& Meyer, 1999; Mcclelland \& Rumelhart, 1981; cf. Lichtheim, 1885). Their representations are thought to be organized via superordinate characteristics, conceivable as nodes (Collins \& Loftus, 1975; Dell, 1986; Levelt et al., 1999; Parks et al., 1992; Rapp \& Goldrick, 2000; Roelofs, 1992), thus constituting a network 
structure referred to as the "mental lexicon." Elementary functions of this system are regarded as the linking of a chosen semantic concept to the best-suited lexical item, its correct grammatical form, and the subsequent phonological processing prior to articulation (Dell, 1986; Levelt, 1999; cf. Indefrey, 2011), the last step of which is likely to interface with the auditory system (Walker \& Hickok, 2015). In terms of conceptualization, it has been proposed that different characteristics of a word (such as the item's function, smell, appearance) are represented within anatomically diverse cortical areas (Crosson, 2013; Hart et al., 2013; Pulvermuller, 1999). With respect to the integration of these semantic features and the lexical selection that follows, it is suspected that, in addition to cortical structures, subcortical structures are also involved (Crosson, Benjamin, \& Levy, 2007; Hart et al., 2013). As opposed to the spread-out semantic network, subsequent phonological processing appears to rely predominantly on temporal areas (Binder et al., 2009; Hickok \& Poeppel, 2007). Activation of the corresponding representations is thought to comprise the coactivation of semantically (Levelt, 1999) and phonologically (Dell, 1986; Foygel \& Dell, 2000) related items. Indeed, the idea of coactivation is supported by facilitatory effects found, for example, in lexical priming studies (e.g., Apfelbaum, Blumstein, \& McMurray, 2011; D. E. Meyer \& Schvaneveldt, 1971), semantic judgment tasks (e.g., Jackson et al., 2015), lexical cluster analysis (e.g., Fitzgerald, 1983; Graesser \& Mandler, 1978; Gruenewald \& Lockhead, 1980; Pollio, 1964; Troyer, Moscovitch, \& Winocur, 1997; Vonberg, Ehlen, Fromm, \& Klostermann, 2014), and phonological neighborhood effects (e.g., Braun et al., 2015; Muller, Dunabeitia, \& Carreiras, 2010). However, it has been proposed that interference among coactivated related items delays the selection of the most appropriate item, as observed, for example, in naming tasks, verbal recall, or verbal fluency (VF) tasks (Bauml, Zellner, \& Vilimek, 2005; Bousfield, Sedgewick, \& Cohen, 1954; Costa et al., 2009; Johnson, Johnson, \& Mark, 1951; McGill, 1963; Raaijmakers \& Shiffrin, 1980; Rohrer, 1996; Rohrer \& Wixted, 1994; Rohrer, Wixted, Salmon, \& Butters, 1995). Since in the following we will focus on word production dynamics in verbal recall and VF tasks, these shall briefly be outlined: Verbal recall tasks require the participants to name as many words as possible from a previously studied list, whereas in VF tasks as many words as possible belonging to a predefined category (semantic VF) or commencing with a given letter (phonemic VF) have to be produced within a set amount of time without prior exposure to a study list (Baldo, Shimamura, Delis, Kramer, \& Kaplan, 2001; Duff, Schoenberg, Scott, \& Adams, 2005; Obeso, Casabona, Bringas, Alvarez, \& Jahanshahi, 2012; for reviews, see J. Henry \& Crawford, 2005; J. D. Henry \& Crawford, 2004a, 2004b; Stein, Luppa, Brahler, Konig, \& Riedel-Heller, 2010).

Beyond the clinical testing that focuses on the total number of words individually produced, the aim of scientific approaches is also to determine underlying cognitive processes. Under this objective, analyzing time courses of word production via curve fitting may serve to provide insight into the dynamics of corresponding brain processes. Two standard functions, still in use today, have therefore been formulated by Bousfield and coworkers in 1944 (Bousfield \& Sedgewick, 1944) and Bousfield et al., 1954 (Bousfield et al., 1954), which are generally recognized for delivering reliable results (Gruenewald \& Lockhead, 1980; Herrmann \& Chaffin, 1976; Herrmann \& Murray, 1979; Herrmann \& Pearle, 1981; D. J. Meyer et al., 2012; Pollio, 1964; Vonberg et al., 2014). By means of a stochastic modeling (see below), their findings suggested an interpretation in terms of lexical search, retrieval, and suppression. On this basis, a large number of further refinements have been elaborated to extend the description and modeling of lexical memory functions (Bauml et al., 2005; Bousfield \& Cohen, 1953a; Herrmann \& Chaffin, 1976; Herrmann \& Murray, 1979; Indow \& Togano, 1970; Kaplan, Carvella, \& Metlay, 1969; Luo, Luk, \& Bialystok, 2010; D. J. Meyer et al., 2012; Raaijmakers \& Shiffrin, 1980; Rhodes \& Turvey, 2007; Rohrer, 1996, 2002; Rohrer \& Wixted, 1994; Rohrer et al., 1995; Shiffrin, 1970; Shiffrin \& Atkinson, 1969; Unsworth, Brewer, \& Spillers, 2013; Unsworth \& Engle, 2007; Young, 2004) (for a review see Wixted \& Rohrer, 1994). In so doing, respective theories have typically linked mathematically identified parameters to presumed language functions.

In this context it is striking that the classical Bousfieldian formulae are hitherto perceived as two competing alternatives. An extension of the exponent-contained explicitly in the hyperbolic and implicitly in the exponential formula - previously posited by Bousfield and coworkers (Bousfield et al., 1954) has, to our knowledge, not yet been conducted. The approach presented here therefore aims to unify both functions and to reevaluate their parameters. In view of the abovedepicted concepts of word retrieval, we will try to relate the newly defined mathematical fitting function to current theories on the lexical network structure.

In the following, we will first provide a critical analysis of the two classical functions introduced by Bousfield and coworkers. Building on this, it will be demonstrated that both formulae are special cases of an overarching family of functions. It will furthermore be shown that this new formula can converge to a logarithmic time course. In the next section, the formula will be applied to data sets from different VF tasks. Afterwards a modeling will be proposed based on existing models. The subsequent discussion addresses possible interpretations of the present findings.

\section{Analysis of the bousfieldian approaches}

This section provides an analysis of the two classical Bousfieldian functions in order to establish the prerequisites 
for their generalization. It exposes an incorrectly defined terminological parameter in the hyperbolic function.

The exponential formula

$n(t)=c \cdot\left(1-e^{-m t}\right)$

was first used by Bousfield and Sedgewick to describe the progression of cumulative word production in respective tasks (Bousfield \& Sedgewick, 1944). Its graph starts at the origin and approaches the asymptote $c$ (i.e., the capacity of an assumed supply) with the rate of growth $m$ to the asymptote. Yet, in recall tasks reinforced by repetition, use of formula (1) led to a larger error of approximation. The authors showed that this weakness was reduced by instead applying the hyperbolic function:

$n(t)=\frac{c^{2} m t}{1+c m t}$,

with $c$ and $m$ designating the same quantities as above (Bousfield et al., 1954). The differential evolutions of the corresponding graphs can be easily illustrated by their distinct half-lives, being $t_{H}=\ln 2 / m$ derived from formula (1) and $t_{H}=1 / \mathrm{cm}$ derived from formula (2). In their data from verbal recall tasks, Bousfield and coworkers found a positive correlation between $c$ and the degree of reinforcement (i.e., the number of repetitions of the study list; in the following labeled as $R$ ) as well as a negative correlation between $m$ and $R$ (see Fig. 1A; cf. Indow \& Togano, 1970).

On this basis they conducted their discussion about possible causes for the distinct behaviors of the respective curves. Despite remarking that participants "produced items more rapidly" (Bousfield et al., 1954, p. 116) as a function of $R$, they missed the opportunity to introduce a concrete quantity to indicate the initial slope in their formula. Instead, the challenging quantity $m$, discussed below, became a central factor. In this context, they also introduced the hypothetical quantities "interference" (cf. Johnson et al., 1951), "efficiency," and "habit strength" into their considerations, which are, however, not stringently comprehensible in mathematical terms, thus leading to contradictions. Of these quantities we shall only retain efficiency (in the following labeled as $E$ ), since it is unambiguously defined as the reciprocal of the half-life.

Their observations also inspired Bousfield and coworkers to discuss different usages of the exponential (1) and the hyperbolic representation (2). They considered applying either function generally to data from both task types despite dissimilar experimental conditions. Thus, without regarding Eq. (1) as exclusively applicable to data from VF tasks and Eq. (2) to verbal recall tasks, they sought to identify the reasons behind the apparent taskspecific advantages. Since they discovered a higher initial word production rate in verbal recall tasks compared to
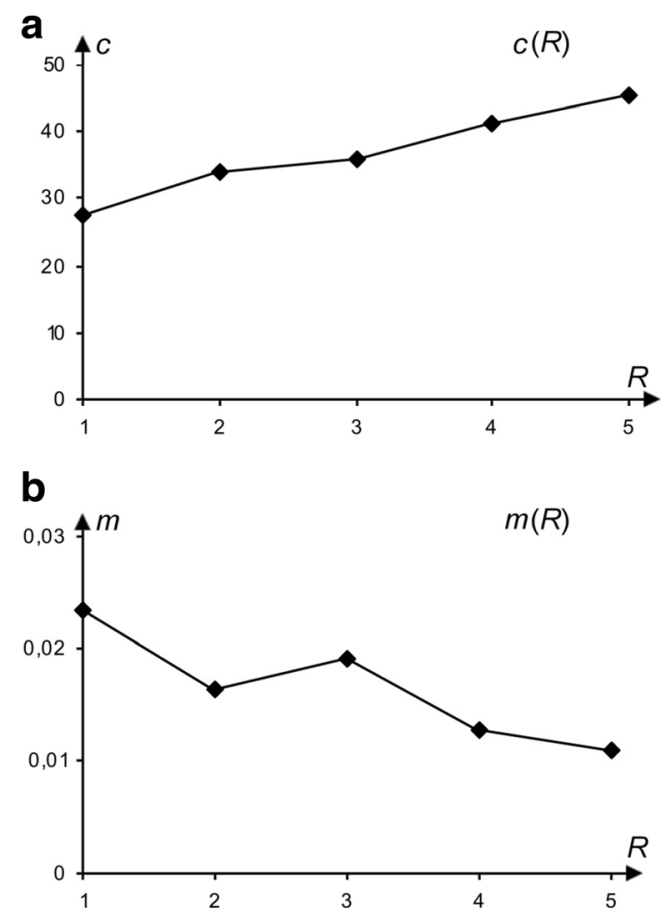

Fig. 1 Reanalyzing the data provided in the data tables by Bousfield et al. (Bousfield, Sedgewick, \& Cohen, 1954) indicates that (a) increased $R$ (number of reinforcements) leads to an almost linear increase of the value of $c$ (number of all uttered words) with a low slope and a high ordinate intercept, and (b) the value of $m$ (with the rate of growth) decreases as $R$ increases

VF tasks, they used this finding, as well as improved fitting results, to endorse the use of Eq. (2) for verbal recall tasks (Bousfield et al., 1954). A critical evaluation of their argumentation will be provided after clarification of the terminology used (see below).

With respect to the differential equations' underlying functions (1) and (2), that is, $n^{\prime}=m \cdot(c-n)^{1}$ and $n^{\prime}=m \cdot(c-n)^{2}$, respectively, the authors addressed the increase of the exponent in the hyperbolic approach (Bousfield et al., 1954). It was apparent that the hyperbolic representation could be derived from the exponential by "switching" the exponent from the discrete value 1 to 2 . Although they mentioned that any other exponent could also have been suitable for optimizing the fit, they did not pursue this notion any further and - to our knowledge - this has not been done thus far. One important reason for this could be that the parameter $m$ (i.e., the approach to the asymptote) was not defined uniformly in both formulae, thus blocking the transformability of the one formula into the other and vice versa. This can be shown most easily by the measuring units of the respective half-lives: in Eq. (1) it follows from $t_{H}=\ln 2 / m$ that the unit of $m$ is $\sec ^{-1}$, whereas in Eq. (2), due to $t_{H}=1 / \mathrm{cm}$, the unit of $m$ is (number $\left.\cdot \mathrm{sec}\right)^{-1}$.

In order to express the two formulae uniformly and to attain a situation where they can be directly related to each other, we 
shall remove $m$ from both formulae. This can be achieved by virtue of the strong negative correlation between $c$ and $m$ found when applying Eq. (1) to empirical data sets (Herrmann \& Murray, 1979; Johnson et al., 1951). A negative correlation is most concisely expressed as inverse proportionality; that is, $m c=r$ is a constant. The meaning of $r$ is elucidated by the first derivation of $n$ given, for example, by $n^{\prime}=m \cdot(c-n)$. Because $n(0)=0$, it is true that $n^{\prime}(0)=m$. $(c-0)=m c=r$ so that $r$ has the meaning of the temporal change rate at the time $t=0$. Graphically, $r$ represents the slope of the graph at its origin (i.e., the initial rate).

In order to change the aforementioned "switch" of the exponent into a "slide," we will first of all substitute the ambiguous parameter $m$ by the terminologically unequivocal parameter $r$. Consequently, formula (1) can be rewritten in a new notation as $n(t)=c \cdot\left(1-e^{-r \cdot t / c}\right)$. According to convention, the exponent can be expressed as $-t / \tau$, with the time constant $\tau=c / r$. So that formula (1) becomes

$n(t)=c \cdot\left(1-e^{-t / \tau}\right)$

As far as we are aware, the initial rate of Eq. (2) has not yet been used to unify both approaches (cf. Herrmann \& Pearle, 1981). Using the differential Eq. of (2), expressed as $n^{\prime}=m \cdot(c-n)^{2}$, it becomes obvious that $r=n^{\prime}(0)=m c^{2}$ as opposed to $r=m c$ in Eq. (1). Inserting the thus derived $r$ into the hyperbolic Eq. (2) yields $n(t)=c r t /(c+r t)$. In order to express the latter as an equivalent of (1a), the time constant $\tau=c / r$ will be used, resulting in:

$n(t)=c \cdot\left(1-\frac{1}{1+t / \tau}\right)$.

Hence, the prerequisite is fulfilled for generalizing the form of the differential equations of the exponential and hyperbolic representations. Substituting $m=r / c$ in $n^{\prime}=m \cdot(c-n)$ yields $n^{\prime}=r / c \cdot(c-n)=r \cdot(1-n / c)$, and substituting $r=n^{\prime}(0)=m c^{2}$ in $n^{\prime}=m \cdot(c-n)^{2}$ yields $n^{\prime}=r / c^{2} \cdot(c-n)^{2}=r \cdot(1-n / c)^{2}$. Accordingly, the derivation $n^{\prime}$ now presents itself in both cases as the product of the initial rate $r$ with the unit number/sec and a unit-less temporal form factor. The corresponding differential Eqs. of (1a) and (2a) are hence:

$n^{\prime}=r \cdot(1-n / c)^{1}$

and:

$n^{\prime}=r \cdot(1-n / c)^{2}$.
Reconsidering the half-lives of the respective functions in the new notation, the exponential function (1a) produces:

$t_{H}=\ln 2 \cdot c / r$

whereas the hyperbolic function (2a) produces:

$t_{H}=1 \cdot c / r$.

After making this clarification and prior to changing the exponent from "switch" to "slide," we are now able to retrospectively and more critically analyze Bousfield and coworkers' motivation for introducing the hyperbolic approximation. In their view, the main reason for doing so was the "initially relatively rapid" (Bousfield et al., 1954, p. 116) rise of the production curves of data obtained from verbal recall tasks in contrast to data from their earlier VF study. In order to derive their hyperbolic approximation they, first, set the cumulative number of produced words to be equal (i.e., $\left.n_{E}=n_{H}=n\right)$, second, assumed an equal supply $c$, and, third, equated $m_{E}$ and $m_{H}$. As a consequence, their differential equations $n_{E}^{\prime}=m_{E} \cdot\left(c-n_{E}\right)$ and $n_{H}^{\prime}=m_{H} \cdot\left(c-n_{H}\right)^{2}$ merged into $n^{\prime}=m \cdot(c-n)$ and $n^{\prime}=m \cdot(c-n)^{2}$, respectively. Because $0 \leq n<c$, it is true that $(c-n) \geq 1$, and therefore $(c-n)^{2} \geq(c-$ $n)$. From this relationship the authors concluded that the hyperbolic course delivered a comparably larger temporal increase, especially for small $t$ values, necessary for their data approximation. Even though the authors made a profound scientific contribution with the introduction of their hyperbolic approach, their argumentation contains several errors: first, $n_{E}(t)$ and $n_{H}(t)$ characterize two distinct time courses that cannot coincide at more than two points (see Fig. 2). Second, due to distinct measuring units of $m$ in either function, $m_{E}=r_{E} / c$ cannot be equated to $m_{H}=r_{E} / c^{2}$. In order to compare the two functions appropriately, their differential Eqs. (1b) $n_{E}^{\prime}=r_{E} \cdot(1-$ $\left.n_{E} / c\right)$ and $(2 \mathrm{~b}) n_{H}^{\prime}=r_{H} \cdot\left(1-n_{H} / c\right)^{2}$ should be used. Because

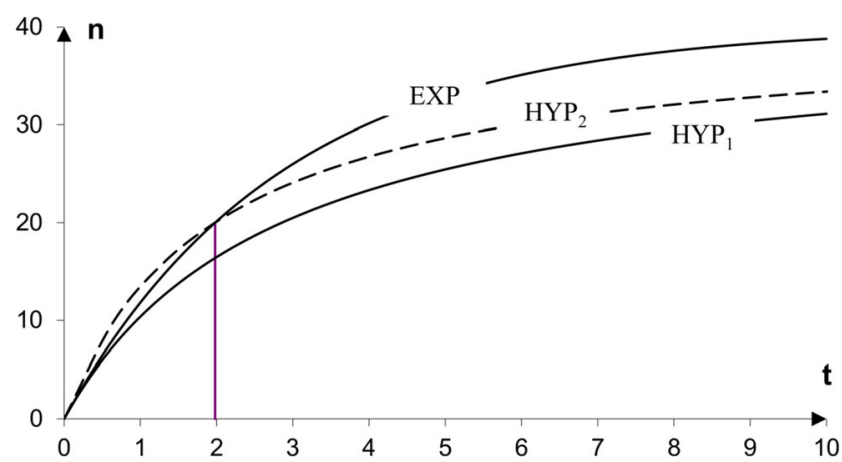

Fig. 2 Shows that an initially steeper slope is not immanent in the hyperbolic course $\left(\mathrm{HYP}_{1}\right)$ if the horizontal asymptote $c$ for an exponential (EXP) and a hyperbolic curve are assumed to be equal. Only if the initial rate is set to a higher value in the hyperbolic $\left(\mathrm{HYP}_{2}\right)$ relative to the exponential case is it possible to realize the assertion made by Bousfield and coworkers (Bousfield et al., 1954). Note. $t=$ time (min), $n=$ number of words 
$(1-n / c) \leq 1$, it is true that $(1-n / c)^{2} \leq(1-n / c)$. This illustrates that for the same $n$ the temporal form factors are related inversely compared to the above relationship. Consequently, for $r_{H}=r_{E}=r$ the hyperbola delivers the opposite of what Bousfield and coworkers had expected of it. The claim that the hyperbola would rise more slowly can only be offset if simultaneously asserting that $r_{H}>r_{E}$. Such a situation occurs (e.g., if identical half-lives of the exponential and hyperbolic time course are requested). In this case, $r_{H} \approx 1.44 r_{E}$ is true, and the initial section of the normally flatter hyperbolic curve will have to be "elevated."

\section{Generalization of the bousfieldian approaches}

In light of this background, the aforementioned third parameter shall now be introduced. To this end, the two differential Eqs. (1b) and (2b) lead to the generalized differential equation:

$n^{\prime}=r \cdot(1-n / c)^{1+\alpha}$.

If $\alpha=0$, then the differential equation will yield (1b) with the corresponding time course of function (1a), and if $\alpha=1$, the differential equation will yield (2b) with the time course of function (2a). Furthermore, the differential Eq. (3) permits a gradual transition of half-lives and efficiencies despite constant values of $r$ and $c$. Moreover, (3) allows the extrapolation to time courses beyond the exponential and hyperbolic ones. This, on the one hand, constitutes a considerable simplification; on the other hand, however, the introduction of a variable exponent makes it necessary to justify this third parameter not only formally but also contextually.

Equation (3) can be solved by separation of variables. Starting with the initial condition, $n(0)=0$ yields a modified power function $n(t)=c \cdot\left[1-(1+\alpha \cdot r \cdot t / c)^{-1 / \alpha}\right]$. Reconsidering the time constant $\tau=c / \alpha r$, the final equation can be expressed as

$n(t)=c \cdot\left[1-\left(\frac{1}{1+t / \tau}\right)^{1 / \alpha}\right]$.

As is immediately apparent with the time course of function (3a), it incorporates both classical functions (1a) and (2a) as special cases. Setting $\alpha=1$ leads directly to $n(t)=c \cdot(1-$ $\left.(1+t / \tau)^{-1}\right)$, that is, the time course of function (2a). Additionally, because $\lim _{\alpha \rightarrow 0}(1+\alpha \cdot x)^{-1 / \alpha}=e^{-x}$ the function produces $n(t)=c \cdot\left(1-e^{-t / \tau}\right)$, that is, the time course of (1a). Our approach produces the half-life $t_{H}=c\left(2^{\alpha}-1\right) / \alpha r$ or, respectively, the efficiency

$E=\frac{1}{t_{H}}=\frac{\alpha}{2^{\alpha}-1} \cdot \frac{r}{c}$.
The first factor $F(\alpha)=\alpha /\left(2^{\alpha}-1\right)$ indicates a decrease in efficiency, if $\alpha$ increases (see Fig. 3), uncoupled from the initial rate $r$ and the parameter $c$.

In view of the aforementioned discussion on efficiency, our approach makes it possible to draw a connection between efficiency and the third parameter in a uniform fashion. For fixed $r$ and $c$, the third parameter permits variable shapes of the time course along with the respective half-lives and efficiencies. Figure 4 represents a family of functions in which a variable half-life $t_{H}$, and thus a variable factor $F$, have been adjusted by means of a variable $\alpha$ at constant values of $r$ and $c$. Consequently, by choosing an appropriate value of $\alpha$ the required half-life $t_{H}$ can be adjusted for arbitrary values of $c$ and $r$. Since the power function (3a) contains its historic predecessors as special cases, its standard deviation regarding any given data set, determined by the method of least squares, must always produce an equal or better fit than approximations performed with either (1a) or (2a).

\section{A limiting function leads to a logarithmic time course}

In more than $70 \%$ of our data acquired from VF tasks (see below), approximation by function (3a) yielded enormously large values of $c$ and $\alpha$. Nonetheless, the approximations were achieved in all cases because the two divergent parameters were always proportionally coupled. In fact, increasing values of $c$ and of $\alpha$ deformed the graph of (3a) in an opposed manner. While an increase in $c$ pulled the graph toward the ordinate, an increase of the shape parameter $\alpha$ precipitated an elongation toward the abscissa. Due to this coupling, the extremes of both parameters were compensated, and the logarithmic time course evolved as a new and not intentionally pursued quality: by inserting the coupling constant $k=c / \alpha$ and determining the limit $\alpha \propto c \rightarrow \infty$, then due to $\lim _{\alpha \rightarrow \infty} a$

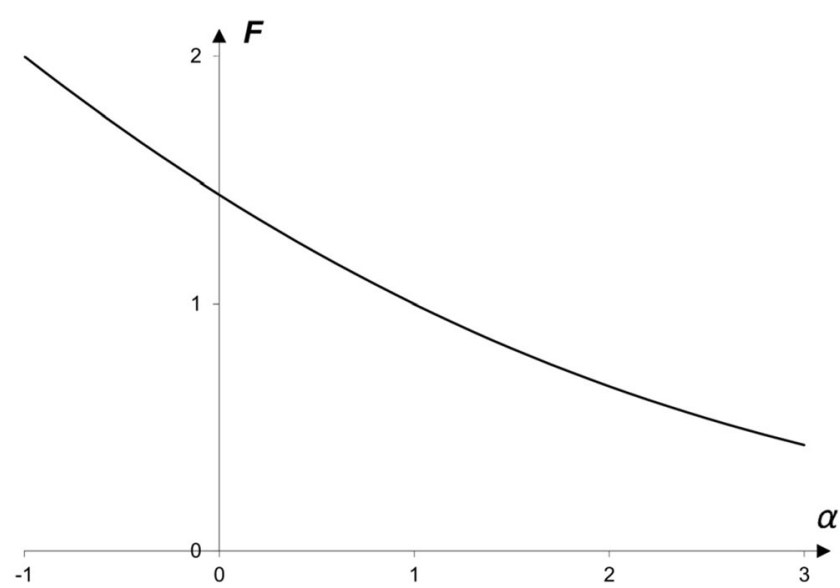

Fig. 3 Factor $F$ is illustrated as a function of the parameter $\alpha$. The graph indicates the function's strictly monotonic decrease 
Fig. 4 A family of functions yielding from Eq. (3a) is shown with the value of $c$ set to 40 words and the initial rate set to $r=1$ word/sec. By inserting different values of $\alpha$, variable values of the half-life $t_{H}$-and therefore of the factor $F$ - have been adjusted. Graphs pertaining to the two classical formulae (1a) or (2a) are represented as special cases if $\alpha$ is set to 0 or 1 , respectively. Note. $t=$ time ( $\mathrm{sec}$ ), $n=$ cumulative number of words

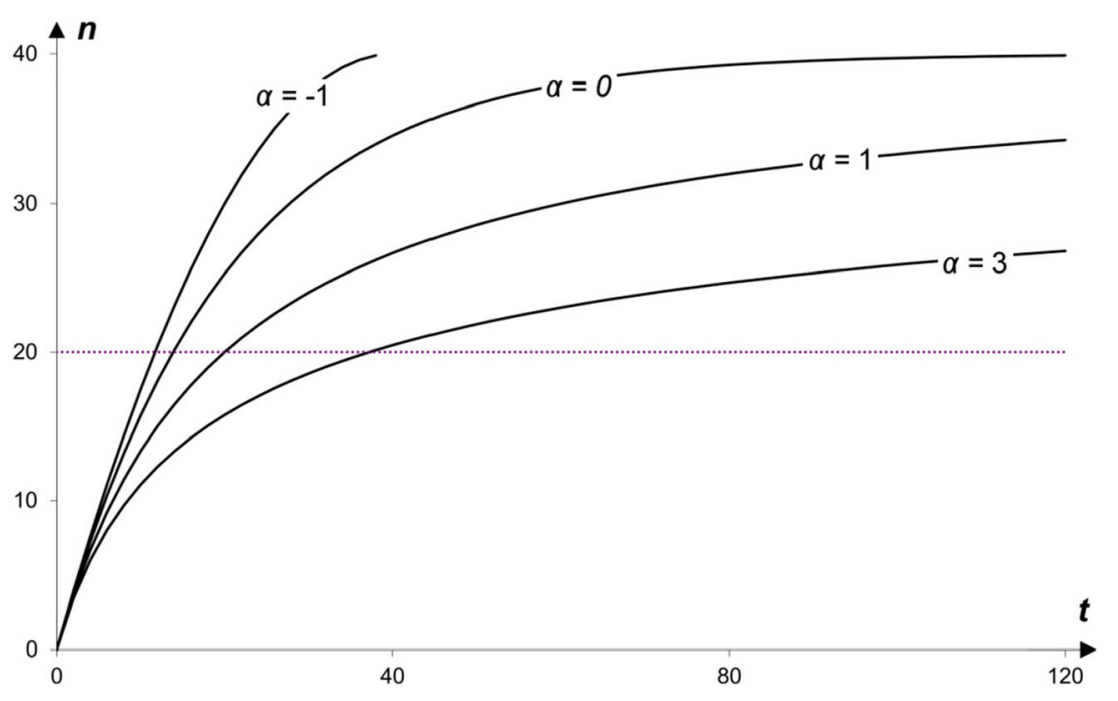

$\left(1-x^{1 / a}\right)=-\ln x$, it can actually be shown that the function (3a) merges into the logarithmic function

$n(t)=k \cdot \ln (1+r t / k)$,

with the derivation

$n^{\prime}=r /(1+r t / k)$.

Since $k$ and $r$ are finite, the half-life $t_{H}=c\left(2^{\alpha}-1\right) /$ $\alpha r=k\left(2^{\alpha}-1\right) / r$ diverges. It follows that the previously defined efficiency of word production approaches zero. In particular, the significance of this special case becomes evident when reanalyzing the data provided by Bousfield and coworkers (Bousfield et al., 1954), as shown below. However, it is worth noting that the time constant remains finite because $\tau=c / \alpha r=k / r$, so that the final form yields the logarithmic time course as the limiting function $n(t)=k \cdot \ln (1+t / \tau)$ (referred to in the following as "logarithmic limit").

\section{Method application}

\section{Curve fitting under application of a hessian matrix}

For reasons of comparison, although the power function $n(t)=c \cdot\left[1-(1+\alpha \cdot r \cdot t / c)^{-1 / \alpha}\right]$ encompasses the other functions, curve fittings will be carried out separately using the power function as well as the two classical functions, that is, the exponential $n(t)=c \cdot\left(1-e^{-r \cdot t / c}\right)$ and hyperbolic $n(t)=c \cdot\left(1-(1+r \cdot t / c)^{-1}\right)$ for curve fitting of all our data sets. Since the logarithmic course $n(t)=k \cdot \ln (1+t / \tau)$ can occur as a possible result of the power function fittings, separate fittings are not needed here.

For fitting any given data set, the sum $Q(c, r)$ or $Q(c, r, \alpha)$ of squared deviations has to be built as well as the first and second partial derivative regarding their two, respectively, three parameters. Since this can be performed analytically (see Appendix 1), the elements of the respective $2 \times 2$ and 3 $\times 3$ Hessian Matrix $H$ and the leading principal minors can also be written in an analytical form. It was therefore possible to discern the corresponding two- or three-dimensional convergence areas without approximation errors. This was determined as the area where $H$ is positive-definite. In so doing, first, a fast running iteration was achieved, and, second, numerical artifacts were strictly avoided even in critical cases. A copy of the program designed to compute and illustrate the two classical functions and the fused Bousfieldian function can be obtained from the authors.

The thus obtained parameter values were compared in the following statistical analysis.

\section{Participants}

Twenty young (11 female and nine male, age $30.20( \pm 5.58)$ years, school education 12.65 ( \pm .49$)$ years, two left-handed) and 23 elderly (seven female and 16 male, age $66.96( \pm 7.71)$ years, school education $10.70( \pm 1.74)$ years, three left-handed) healthy participants were included in the study. All participants were native German speakers, had no history of neurological and psychiatric diseases, and did not receive any centrally acting drugs. They gave written informed consent to the study protocol approved by the Ethics Committee of the Charité (protocol number EA2/ 047/ 10).

\section{Verbal fluency (VF) task}

All participants performed the German standard version of a verbal fluency task (Regensburger Wortfluessigkeits-Test; Aschenbrenner, Tucha, \& Lange, 2000). They were asked to produce as many German words as possible in 120 seconds 
under four different task conditions: (i) Semantic nonalternating (vegetables), (ii) Phonemic nonalternating (words starting with "s"), (iii) Semantic alternating (animals/ pieces of furniture), and (iv) Phonemic alternating (words starting with "g"/"cr"). Repetitions of entire words or word stems as well as proper names were not allowed. But since these are also considered to be informative in terms of underlying cognitive processes (Troyer et al., 1997), they were included in the analysis. Metacomments (e.g., "I don't know any more words") were excluded. The samples were digitally recorded (computer software Audacity 1.3.13-beta). The starting and ending point of each uttered word was determined acoustically by means of the audio track at a temporal resolution of $1 \mathrm{~ms}$.

\section{Statistical analysis}

The statistical analyses were carried out using SPSS version 22.0 and GPower version 3.1.9.2 (Faul, Erdfelder, Lang, \& Buchner, 2007). Kolmogorov-Smirnov tests for normal distribution were carried out prior to the following analyses. Bonferroni corrections were used for all multiple comparisons.

To estimate differences in the accuracy of fit between the three functions, an analysis of variance (ANOVA) for repeated measures was performed for the mean values of $\sigma$ from all four tasks (data were normally distributed). The following analyses were carried out only for data yielded by the power function developed here (3a): separate ANOVAS for repeated measures for the normally distributed parameters "initial rate" $(r)$ and "total number of words" $(N)$ each with the withinsubject-factor "task" (four levels) and the between-subjectfactor "group" (i.e., old vs. young) as well as the Friedman test for the nonnormally distributed parameter $k(k=c / \alpha)$.

\section{Application of the Bayesian information criterion (BIC)}

The Bayesian information criterion (BIC) has been formulated to enable a numerical evaluation of the balance between effort and goodness of fit (i.e., the gain of information resulting from data analysis; Schwarz, 1978; cf. Kahana, Zhou, Geller, \& Sekuler, 2007; Myung \& Pitt, 1997), as initially demanded by Occam (Occam's Razor, 2015). If the minimal standard deviation achievable $(\sigma)$ is applied as the target parameter for goodness of fit the criterion is expressible as $B I C=\kappa \ln N+N \ln \sigma^{2}$, with $N$ being the number of all uttered words and $\kappa$ the number of fitting parameters. The first summand expresses the effort and increases with an increasing number of variables, whereas the second summandrepresenting goodness of fit-decreases if fit is improved. Accordingly, smaller BIC values indicate superiority.

In the present study, BIC was used to assess the informative value of the parameter extension from $\kappa=2$ (in the exponential and hyperbolic function) to $\kappa=3$ (in the power function). For this purpose, difference values of the power and the exponential function $\triangle B I C_{P, E}=\left(B I C_{P o w e r}-B I C_{E X P}\right)$ and between the power and the hyperbolic function $\triangle B I C_{P}$, ${ }_{H}=\left(B I C_{\text {Power }}-B I C_{H Y P}\right)$ were first determined for each data set. Thereafter, the mean values of the respective difference values were computed. The occurrence of negative mean difference values indicated a fulfilling of the BIC requirements by the power function.

\section{Results}

\section{Curve fitting}

All data sets could be fitted successfully by the three functions. Of the power functions, $78.49 \%$ were logarithmic functions - which were special and unexpected cases - with both $c$ and $\alpha$ approaching infinity. It is important to mention that the relationship between $c$ and $\alpha$ was found to be constant, expressed as $k=c / \alpha$.

\section{Statistical analysis}

As expected from our general approach, accuracy of fit differed significantly between the three functions, $\mathrm{F}(2,41)=$ $46.55, p<.001$, with best fits for power $(\sigma=.77 \pm .16)$, followed by hyperbolic $(\sigma=.84 \pm .16)$ and exponential $(\sigma=$ $.92 \pm .18$ ) functions (Cohen's effect size $f=.35$; statistical power $=.94)$. Post hoc analysis indicated significant differences for all comparisons (power vs. hyperbolic, $p=.001$; power vs. exponential, $p<.001$; hyperbolic vs. exponential, $p<.001)$.

In terms of the power function, $r$ differed significantly between the four task conditions, $F(3,39)=15.72, p<.001$, and the two age groups, $F(1,41)=6.87, p=.012$, but there was no significant interaction between task condition * age group. The initial rate was highest in Semantic alternating $(r=.67$ $\pm .22)$, followed by Semantic nonalternating $(r=.63 \pm .27)$, Phonemic nonalternating $(r=.52 \pm .19)$, and Phonemic alternating tasks $(r=.44 \pm .17$; Cohen's effect size $f=.15$; statistical power $=.32$ ). Post hoc analysis indicated significant differences in the initial rates between Semantic nonalternating and Phonemic alternating $(p=.001)$, Semantic alternating and Phonemic nonalternating ( $p=$ $.001)$, as well as between Semantic alternating and Phonemic alternating tasks $(p<.001)$ across both age groups.

In line with varying demands imposed by the task condition, the value of $N$ differed significantly between the four task conditions, $\mathrm{F}(3,39)=17.90, p<.001$. There was neither a significant difference between the two age groups nor a significant interaction between task condition $*$ age group. Largest values were found in Semantic alternating ( $N=$ 
$27.60 \pm 5.05)$, and Phonemic nonalternating tasks $(N=27.30$ $\pm 9.23)$, followed by Phonemic alternating $(n=24.33 \pm 6.73)$ and, finally, Semantic nonalternating tasks $(N=20.84 \pm 4.73$; Cohen's effect size $f=.38$; statistical power $=.96$ ). Post hoc analysis indicated significant differences between Semantic nonalternating and Semantic alternating $(p<.001)$, Phonemic nonalternating $(p<.001)$ and Phonemic alternating tasks $(p=.005)$, between Semantic alternating and Phonemic nonalternating ( $p=.004)$, as well as between Phonemic nonalternating and Phonemic alternating tasks ( $p$ $=.015)$ across both age groups.

The nonnormally distributed parameter coupling constant ( $k$; defined as $c / \alpha$ ) did not vary significantly between the task conditions or age groups, and no significant interaction was found, median (quartile 1; 3): 18.57 (11.69; 31.46) across all tasks and participants.

\section{Evaluation of BIC}

Mean differences of $\mathrm{BIC}$ values were negative if comparing the power function to either the exponential $\left(\Delta B I C_{p, e}=\right.$ -4.96; corresponding to an improvement of $\sim 161 \%$ ) or the hyperbolic function $\left(\triangle B I C_{p, h}=-.41\right.$; corresponding to an improvement of $\sim 19 \%$ ). The requirements of the criterion were therefore met in both cases suggesting a higher informative value of the power function.

\section{Modeling}

In order to interpret the time course and respective parameters of the new generalized function (3a) and its logarithmic limit (5), we attempt to develop models based on those that have already been formulated for the two special cases, that is, the exponential (1a) and hyperbolic (2a) function.

\section{Modeling the time course of the exponential function (1a) according to a simple sampling-with-replacement model}

A modeling for the exponential function shall be designed using a simple, probabilistic approach. This view establishes the basis for the model's extension delineated in the following sections. It originates from the stochastic interpretation of the exponential time course as a discrete (i.e., noncontinuous) random retrieval process or a special type of Furry-Yule process (D. Albert, 1968; McGill, 1963), in which the underlying differential Eq. (1b) is approximated by a difference equation. According to this sampling-with-replacement model, all items (here, words) are assumed to have the same probability of being sampled from consecutive "search sets" that are to be randomly activated by a task-specific cue at a steady rate (cf. Rohrer, 1996; Shiffrin, 1970; Wixted \& Rohrer, 1994). Each word produced would be "replaced" in the search set and regain the same probability of - now erroneously-being sampled again. The concept of the time course of the exponential function was illustrated by the probabilistic analog model of drawing and replacing balls from an urn (Herrmann \& Pearle, 1981; McGill, 1963; Wixted \& Rohrer, 1994). It was emphasized that the simple sampling-withreplacement model unexpectedly provided many feasible starting points for further developments (Wixted \& Rohrer, 1994). Since this will also constitute the starting point of our approach, we will first repeat the derivation of (1a) using the simplest possible probabilistic model in order to subsequently develop the targeted model extension in a transparent fashion.

Using the example of VF tasks, one could reasonably formulate that an urn was initially filled with only white balls, representing a one-dimensional lexical list of all single suitable words. Their number $c$ would correspond to the capacity of the urn. Red balls, on the other hand, would represent already produced words, the repetition of which would constitute an error. Initially, the urn would therefore contain zero red balls. This simplified model purposely ignores other types of errors not pertaining to word repetition. It furthermore assumes independence of the draws. According to the sampling-with-replacement model, on producing the first correct word, the corresponding white ball would be replaced by a red one. Consequently, the content would maintain a constant value $c$. After the first draw, the urn would necessarily contain $c-1$ white balls and one red ball. In general, the drawing of either a white or a red ball is set to require the same "elementary process duration" $\Delta t$ (cf. Kaplan et al., 1969) due to $\Delta n / \Delta t=1 / \Delta t=r$ leading to

$\Delta t=1 / r$

In the event a white ball is drawn, $\Delta t$ would be needed for the sampling and retrieving of a correct word, whereas if a red ball is drawn, $\Delta t$ would be needed for the sampling and suppressing of an incorrect word. The elementary process duration would thus be the reciprocal of the initial rate $r$. This underlines that the substitution of the Bousfieldian $m$ by the initial rate $r=m c$ was reasonable, because in the model the parameter $r$ is now given a direct meaning (i.e., the reciprocal of the elementary process duration). In case of the first word, the elementary process duration $\Delta t$, the "interresponse time" (IRT) $\tau_{1}$, and the "cumulative IRTs" $t_{1}$ would coincide as $t_{1}=\tau_{1}=\Delta t$. Before the retrieval of the $n$th white ball, the urn would contain $c-(n-1)$ white and $n-1$ red balls so that, according to Laplace's principle, the probability of drawing the $n$th white ball would be reduced to $p_{n}=(c-(n-1)) / c$. A stepwise linear decrease of the probabilities $p_{1}=1-0 / c, p_{2}=$ $1-1 / c, p_{3}=1-2 / c$, and so forth would thus result, and an increasing number of false draws would have to be expected, each requiring the duration $\Delta t$. It is worth noting here that the comparison of $p_{n+1}=1-n / c$ to the differential Eq. (1b), that is, 
$n^{\prime}=r \cdot(1-n / c)$ delivers a generally valid relationship between the probabilities of a sampling-with-replacement model and the slopes of the respective fitting curves. It is true that

$p_{n+1} \approx n^{\prime} / r$.

Assume that the $w$ th draw would deliver the $n$th white ball due to previous $w-1$ draws of red balls. Since the model presumes a replacement of red balls, the number $w-1$ of false draws could lie between zero and infinity. Consequently, the probability of attaining the $n$th white ball with the $w$ th draw would be $P_{n}(w)=q_{n}^{w-1} \cdot p_{n}$ with $q_{n}=1-p_{n}$. The average number of draws necessary for attaining the $n$th white ball is therefore given by the expected value $\bar{w}_{n}=\sum_{w=1}^{\infty} w \cdot P_{n}(w)$. Inserting the above probability $P_{n}(w)$ and transformation leads to

$\bar{w}_{n}=1 / p_{n}$.

Accordingly, the average number of draws required to attain the $n$th white ball is equal to the reciprocal of the probability of attaining it on the first draw.

The corresponding standard deviation can be assessed by a similar approach, yielding $\sigma_{n}=\sqrt{1-p_{n}} / p_{n}$. The IRT $\tau_{n}$, for drawing the $n$th white ball is therefore $\tau_{n}=\bar{w}_{n} \Delta t=\Delta t / p_{n}$ $=\Delta t \cdot c /\left[\left(c^{-}(n-1)\right]\right.$. Cumulation of IRTs needed for retrieval of the $n$th white ball leads to the following harmonic partial series:

$$
\begin{aligned}
t \approx t_{n} & =\sum_{i=1}^{n} \tau_{i}=\Delta t\left(\frac{1}{p_{1}}+\frac{1}{p_{2}}+\ldots+\frac{1}{p_{n}}\right) \\
& =\Delta t\left(\frac{c}{c}+\frac{c}{c-1}+\ldots+\frac{c}{c-(n-1)}\right) .
\end{aligned}
$$

Computation of the sum yields the cumulative IRT, that is, $t \approx c \Delta t[\ln c-\ln (c-n)]$. According to the above identification of $\Delta t$ as the reciprocal of the initial slope, $1 / r$ - see formula (6) - (7a) presents itself as $t \approx(c / r) \cdot \ln c /(c-n)$. Transposition delivers $n(t)=c \cdot\left(1-e^{-r \cdot t / c}\right)$, that is, formula (1a).

Figure 5 illustrates the cumulative IRTs of the series of produced words as provided by the simple sampling-withreplacement model. It is worth noting that the time course of function (1a) is represented by equivalent parameter values. Thus, the sampling-with-replacement model provides a mechanism that leads to the formula presented by Bousfield and Sedgewick (1944).

\section{Modeling the time course of the hyperbolic function (2a) according to an extended sampling-with-replacement model}

In the following section we will derive an analogous stochastic model for the hyperbolic function. It will be shown that the approach proposes pairs of items rather than single items as constituents of the presumed supply. This constitutes the premise for interpreting the here presented released exponent as an indicator of word complexes.

To our knowledge, no comparable sampling-withreplacement model has yet been formulated for the hyperbolic function. According to the above considerations, the respective probabilities $p_{n+1}$ can be determined by combining (6a), that is, $p_{n+1} \approx n^{\prime} / r$, with the differential equation of the hyperbolic curve (2b), that is, $n^{\prime}=r \cdot(1-n / c)^{2}$. This delivers

$p_{n+1}=(1-n / c)^{2}=(c-n)^{2} / c^{2}$.

The interpretation of the probabilities $p_{1}=c^{2} / c^{2}$, $p_{2}=(c-1)^{2} / c^{2}, p_{3}=(c-2)^{2} / c^{2}$, and so forth provides the required model: Compared to the simple model, these squared probabilities would in fact result if the supply consisted of
Fig. 5 Illustration of the cumulative IRTs $\left(t_{n}\right.$ on the abscissa) of a series of produced words ( $n$ on the ordinate; here set to 20) as would be expected from the classical sampling-withreplacement model. Already at this relatively small value of $n$, the time course provides a good approximation of the exponential function (1a). Note. $t=$ time (sec), $n=$ cumulative number of words

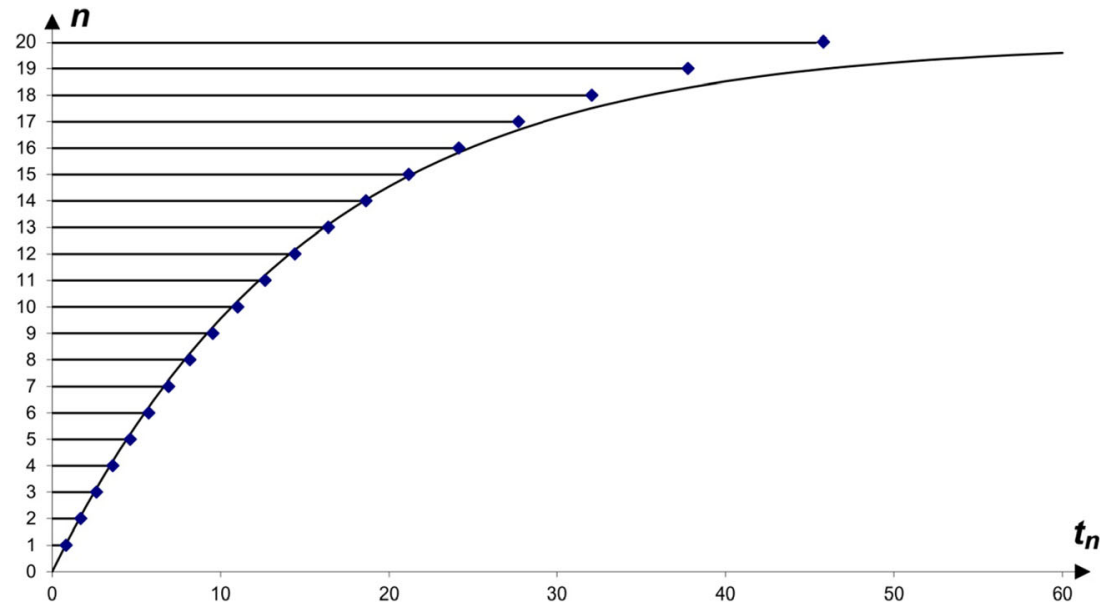


pairs rather than single items. Consequently, on choosing any white ball, all pairs pertaining to the respective ball would become red. Therefore, before the draw of the $\nu$ th white ball, the supply would consist of $[c-(\nu-1)]^{2}$ white pairs (i.e., "favorable objects") while the rest would be red. Again, according to Laplace's principle, the probability of retrieval is given by $p_{v}=[c-(\nu-1)]^{2} / c^{2}$ and the respective IRT by $\tau_{v}=\Delta t / p_{v}$.

Since the partial number of nonordered pairs is essentially half the number of ordered pairs, the same hyperbolic course would be obtained if non-ordered pairs are presumed to be the urn's content.

Compared to the single-item model, the respective standard deviations $\sigma_{n}=\sqrt{1-p_{n} / p_{n}}$ are enlarged, entailing strongly overlapping variance intervals of the IRTs of both classical formulae. This may further explain why dichotomous modeling has not been questioned before.

Figure 6 indicates the magnification of the noncumulative IRTs and their statistical variances in the case of an urn containing ten balls. Quite distinctly, for $\alpha=0$ ( $\square$ in Fig. 6) IRTs increase from $\tau_{1}=\Delta t$ to $\tau_{10}=10 \Delta t$ and for $\alpha=1$ ( $\Delta$ in Fig. 6) they increase from $\tau_{1}=\Delta t$ to $\tau_{10}=100 \Delta t$.

This simple modeling is to some extent similar to a twostage process expressed stochastically by the double urn model proposed by Herrmann and Pearle (1981). In a nutshell, they suggested a retrieval and assessment of balls from two consecutive urns, with those from the first urn containing word fields and those from the second - drawn according to the selected field - containing proper words. Our model, on the other hand, assumes connections between the objects contained in a single urn.

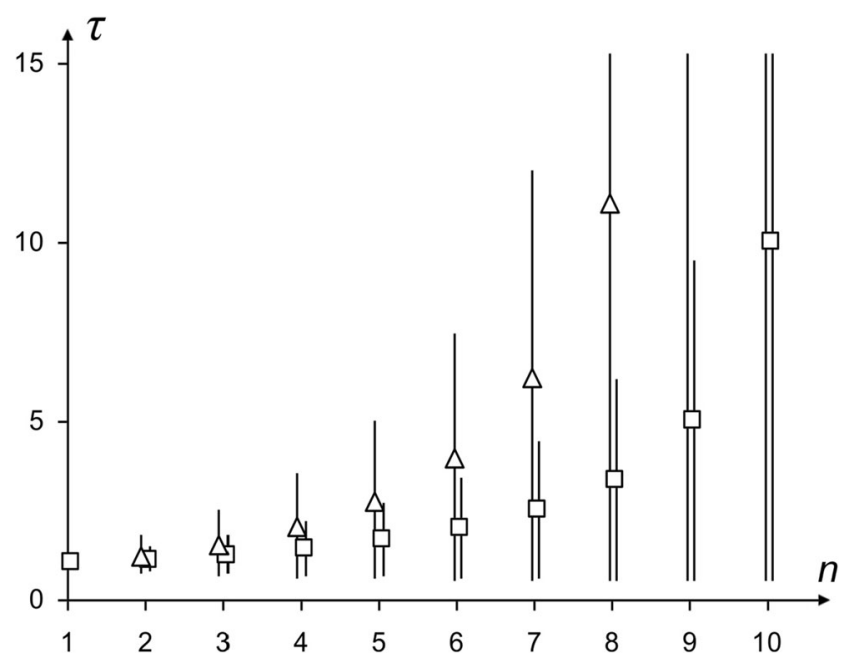

Fig. 6 Illustration of the noncumulative interresponse times (IRTs; $\tau$ on the ordinate) for an urn, which is set to contain 10 balls ( $n$ on the abscissa), with values of $\alpha$ being set to 0 (mean values represented by $\square$ ) or 1 (mean values represented by $\Delta$ ). In the course of drawing the first to tenth ball, IRTs increase from $\tau_{1}=\Delta t$ to $\tau_{10}=10 \Delta t$ in the former case, and from $\tau_{1}=\Delta t$ to $\tau_{10}=100 \Delta t$ in the latter. Vertical lines indicate their overlapping statistical variances
Mathematically speaking, a pair can be considered a tuple of two connected elements, therefore making the tuple two-dimensional. Analogously, triples are threedimensional tuples, and so forth. Thus, with respect to the hyperbolic function, if word pairs rather than single words are assumed - as with the exponential function-it is possible to interpret the data as an extension of the tuple dimension from 1 to 2 if $\alpha$ is increased from 0 to 1. This again would constitute the simplest case of word complexes making up the supply, and indicates an interpretation of $\alpha$ as the degree of connectedness.

Initially no model was formulated for the hyperbolic approach since it had been developed primarily to minimize the error of approximation in the event of reinforced verbal recall tasks. (Bousfield et al., 1954) That said, the authors viewed the concept of lexical interference between the thus reinforced lexical items as a likely cause for the hyperbolic shape.

\section{Modeling the time course of the power function (3a) according to an extended sampling-with-replacement model}

Just as it cannot be assumed that a word supply or mental lexicon actually consists of a one-dimensional list of single words, it also cannot be expected to contain only pairs. But since simple models never aim to replicate reality exactly, but instead serve to mark out its essential aspects, the above considerations can help to extend the idea of connectedness.

The probabilities of the modeling are now given by the combination of (6a), that is, $p_{n+1} \approx n^{\prime} / r$, and (3), i.e. $n^{\prime}=r \cdot(1-$ $n / c)^{1+\alpha}$, as

$p_{n+1}=(1-n / c)^{1+\alpha}=(c-n)^{1+\alpha} / c^{1+\alpha}$.

Since $\alpha$ is not necessarily an integer, an interpretation of the probabilities in analogy to the "Modeling the Time Course of the Hyperbolic Function (2a) According to an Extended Sampling-With-Replacement Model" section indicates that sampling-with-replacement fitting of the empirical data necessarily leads to the assumption of a fractal preorganization of word complexes.

Note that the corresponding noncumulative density function $n^{\prime}(t) \propto(c+r \alpha t)^{-(1+1 / \alpha)}$ of Eq. (3) is a translated power function, as previously stated in a comparable context (Wixted \& Ebbesen, 1991).

\section{Modeling the time course of the logarithmic function (5) according to an extended sampling-with-replacement model}

In this section it will be demonstrated that the logarithmic time course can also be reproduced stochastically, and that its modeling suggests a holistic structure of the lexical storage. 
Starting with the differential equation

$n^{\prime}=d n / d t=r e^{-n / k}$.

of the time course (5), that is, $n(t)=k \cdot \ln (1+r t / k)$, a samplingwith-replacement model can also be reconstructed here. Utilization of $p_{n+1}=n^{\prime} / r$ (6a) again delivers the model's probabilities:

$p_{n+1}=\left(e^{-1 / k}\right)^{n}$.

As long as $k>1$, they develop according to a decreasing geometric sequence. This shall be illustrated by the example of $k \approx 20$, a value commonly observed in our empirical data (see below). Assuming this value, the probability of producing a suitable word decreases according to $p_{n} \approx 0,95^{n}$, and the respective IRTs increase in a realistic manner according to $\tau_{n}=\Delta t / p_{n} \approx 1,05^{n} \cdot \Delta t$. As far as the logarithmic time course is concerned, the actual question then becomes: Which type of organizational form of the stored lexical items can be assumed to cause the IRTs to qualitatively follow a geometric sequence? To answer this question, we must determine the number of all "favorable objects" and the "total number of all possible objects" so that their ratio produces the probability $p_{n+1}$. Here, $\left(e^{1 / k}\right)^{N-n}$ can be considered to be the number of all favorable objects and $\left(e^{1 / k}\right)^{N}$ to be the total number of all possible objects, since their ratio $\left(e^{1 / k}\right)^{N-n} /\left(e^{1 / k}\right)^{N}$ yields $p_{n+}$ ${ }_{1}=\left(e^{-1 / k}\right)^{n}$; where $N$ is set as the number of words available (e.g., during a 120-second testing interval). What possible word conglomerations might make up the supply now? An idea is provided by the special case $p_{n+1}=0,5^{n}$, which yields $\bar{k}=1 / \ln 2 \approx 1,44$. The total number of all possible objects is in this case given by $\left(e^{1 / \bar{k}}\right)^{N}=2^{N}$. This type of amount is known to be the number of all nonordered tuples that can be constructed from $N$ elements (i.e., singles, doubles, triples, quadruples) and even the empty tuple. Subtraction of one element lowers the number of tuples to $2^{N-1}$ (i.e., its initial number is halved). The series of tuples hence provides the requested decreasing geometric series, in this case with the base .5. According to Shannon (1948) this reflects the amount of information to be $N$ binary digits.

Transferred to words produced in VF tasks, this would ideally presume the mental lexicon to be holistically preformatted with all possible conglomerations of (pre-)lexical items. A sampling with replacement could thus be modeled by assuming that with each word produced, all tuples pertaining to the particular word would be replaced and require suppression. The fact that in our cohort $k$ values were about 20 , and thus markedly larger than $\bar{k}=1 / \ln 2 \approx 1,44$, corresponds to a comparably slower reduction of the probabilities. In the proposed model, this would result from an incomplete tuple formation.

\section{Possible causes for the trend toward a logarithmic time course}

Similarities between characteristics of the time courses analyzed here and those of natural networks will now be discussed. Their investigation demonstrates that the logarithmic time course is the most relevant for illustrating a natural network structure.

The proposed models offer potential ways for elucidating the mechanisms underlying word production during VF task performance. However, they do not provide an explanation for the high rate of logarithmic time courses among our data sets. To better understand this aspect, we begin by examining the density distributions $\rho$ of our cumulated distributions $n(t)$. A density distribution indicates how many words $(\Delta n)$ are produced per time interval $\Delta t$; it is therefore given by $\Delta n / \Delta t$. For the continuous function $n(t)$, the empirically measured IRTs merge into the continuous density distribution $\rho(t)=n^{\prime}(t)$, such that $\rho(t)$ presents itself graphically as the slope of $n(t)$.

All of the assessed cumulated distributions had decreasing positive slopes throughout, thus approaching zero asymptotically. They were therefore reminiscent of density distributions related to the nodal degrees $g$ found within networks (Newman, 2005; cf. Pareto, 1897). Self-similarity is a specification of naturally grown networks. The autocorrelation $\gamma$ of the corresponding density distribution serves as an indicator of the degree of self-similarity. It is obtained by calculating the correlation between the distribution function $\rho(g)$ and the rescaled distribution function $\rho(p \cdot g)$, with $p$ as the rescaling factor. Power functions of the type $\rho(g) \propto g^{-\beta}$ are known to be $100 \%$ self-similar. The course of autocorrelation is thus constantly equal to 1 independent of $p$. But neither the BarabásiAlbert network model (R. Albert \& Barabasi, 2002) nor real networks follow exactly a power law, so that $\gamma(p)$ decreases from its initial value of 1 to 0 as the rescaling factor increases toward $p \rightarrow \infty$. The slower the decrease, the more "natural" the network. Although nodes are distributed spatially, their network character is not related to space but rather to the characteristics of their frequency distribution. In analogy, we used the autocorrelation behavior of our times courses' density distributions to assess their "naturality." Whereas autocorrelation of the general power function had to be determined numerically, corresponding formulae were derived for the exponential, hyperbolic, and logarithmic course. Respective data are provided in Appendix 3.

Figure 7 illustrates the increasing tendency toward autocorrelation with lowest values in relation to the exponential, followed by the hyperbolic and the power function (with $\alpha$ arbitrarily set to 3 ), and highest values in relation to the logarithmic function. As required, these depend solely on the characters of the functions, but not on their specific parameter values. 
Fig. 7 Illustration of the degree of autocorrelation $(\gamma)$ of the respective density distributions of the exponential (EXP), hyperbolic (HYP), power (Power, here, $\alpha=3$ ), and logarithmic (LOG) functions as a function of the rescaling factor $p$. The logarithmic time course exhibits the strongest autocorrelation

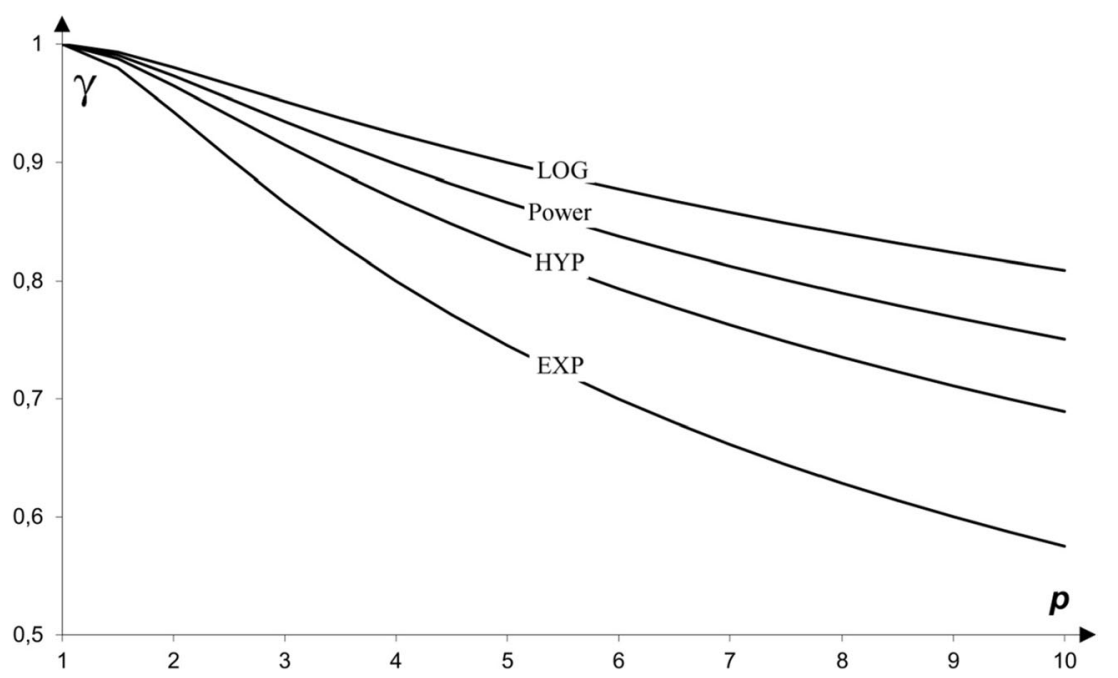

\section{Illustration of a tuple activation and suppression (TAS) model}

In the following, the modeling of the generalized Eq. (3a) shall first be illustrated by a simple numerical example and thereafter be transferred to hypothesized word production processes during VF.

As derived above, in our modeling $\alpha$ can be interpreted as an indicator of the tuple size, thus representing the degree of connectedness within conglomerates of (pre-)lexical items. Regarding the function of the language network in the process of VF performance, we believe that two task rules gain special importance: I. Words must pertain to the given semantic or phonemic superordinate criterion; II. No repetitions of entire words or word stems are allowed. The first rule implies the suppression of all associations not belonging to the task criterion; the second can be interpreted as the necessity to suppress reactivation of each word after its production, as expressed by the sampling-with-replacement model, and implies the principle of interference effects. Assuming a tuple structure, word production would therefore not only require suppression of the respective item but also of all of its connections. Although our $\alpha$ values indicate variable and highly dimensional relations, for the sake of clarity, the current simple model illustrates the (unlikely) case of tuples of two, that is, doubles, representing the special case of the hyperbolic function. The tuple size is therefore exactly equal to the number of connections between each uttered word and all uttered words including auto-relationships. Since this approach can only take into account those words $(N)$ that were actually produced within the given amount of time, no assumptions can be made about the number or the kind of relationships to unuttered lexical items. However, a definite relationship between all produced words is generated by the task criterion (e.g., "types of animals," "girls' names," "words starting with the letter F"), thus indicating a superordinate feature that can serve to illustrate the least degree of connectedness. A corresponding, simple model is illustrated in Fig. 8, which assumes the task criterion "words starting with the letter S" and a comparably small number of produced words $(N=6)$.

Before retrieval of the first word (see Fig. 8A), six appropriate words could theoretically be activated, each of which would be assumed to have six relationships (i.e., five relationships with other words and one auto-relationship, yielding a total of $N^{(1+\alpha)}=36$ favorable relationships). After its production, the first word would have to be suppressed, following Rule II. However, again due to network co-activation, all of its relationships, that is, $N^{(1+\alpha)}-(N-1)^{(1+\alpha)}=11$, would have to be suppressed (see Fig. 8B). This stepwise procedure would be repeated until all words and relationships would be suppressed (see Fig. 8C-F). As is evident, the effort to suppress words and their relationships would increase at a rate greater than the suppression of single interfering words. This sequence would yield the course of the power function with $\alpha=1$. The model becomes more realistic if, instead of pairs, more complex conglomerations are considered (i.e., $\alpha>1$ ). Likewise, if $\alpha=0$, as is the case with the exponential function, the model would only take auto-relationships into account, which can be understood as a one-dimensional lexical list of words.

If generalized and transferred to the assumed processes behind VF, this idea suggests that all eventually expressed words would be theoretically accessible prior to the selection of the first word from the mental lexicon. However, within a natural network, the probabilities of being activated should differ depending on their habit strengths. The process of activation and selection of (pre-)lexical items can be understood as enhancing the activity of a chosen entry while - to a lesser degree - also enhancing the activity of closely related alternatives, followed by a suppression of the nonselected competing items, which, as a whole, strengthens the signal-to-noise ratio (Crosson et al., 2007). Accordingly, it is possible to interpret that the relationships 
a

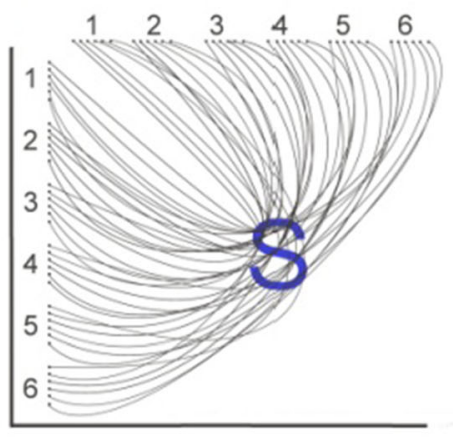

b

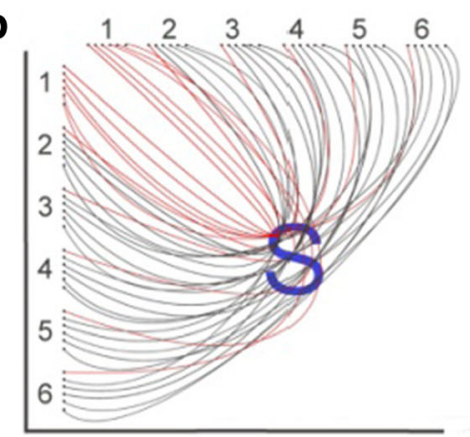

C

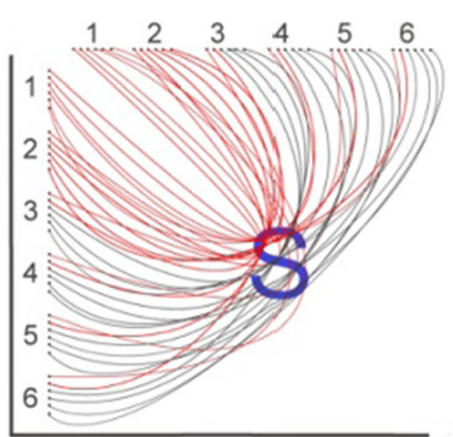

Fig. 8 Illustration of the probabilistic tuple activation and suppression (TAS) model by the simplest case (i.e., ordered pairs), which would be assumed if the shape parameter $\alpha$ Eq. 1 . The number of words produced within a given testing interval is set to $N=6$. Since the value of $\alpha+1$ expresses the dimension of connectedness, each word is illustrated twice (i.e., in the column and row), such that all connections can be indicated by connecting lines. It is proposed that all words are related to each other at least via their initial letter (i.e., the test condition. here, the letter "S"). Black lines represent favorable relationships that can be activated; red lines represent relationships to be suppressed. Auto-relationships represent the selected words. (Color figure online.). A: Before naming

between the distinct items illustrated here are the result of coactivation, whereas auto-relationships correspond to the selection of the target item.

Coactivation is generally thought to occur both within the semantic and the phonemic domains (Braun et al., 2015; Jackson et al., 2015; Muller et al., 2010). Furthermore, since phonological processing is preceded by semantic activation and selection (Costa et al., 2009; Levelt, 1999; Walker \& Hickok, 2015), and activation among phonemically related
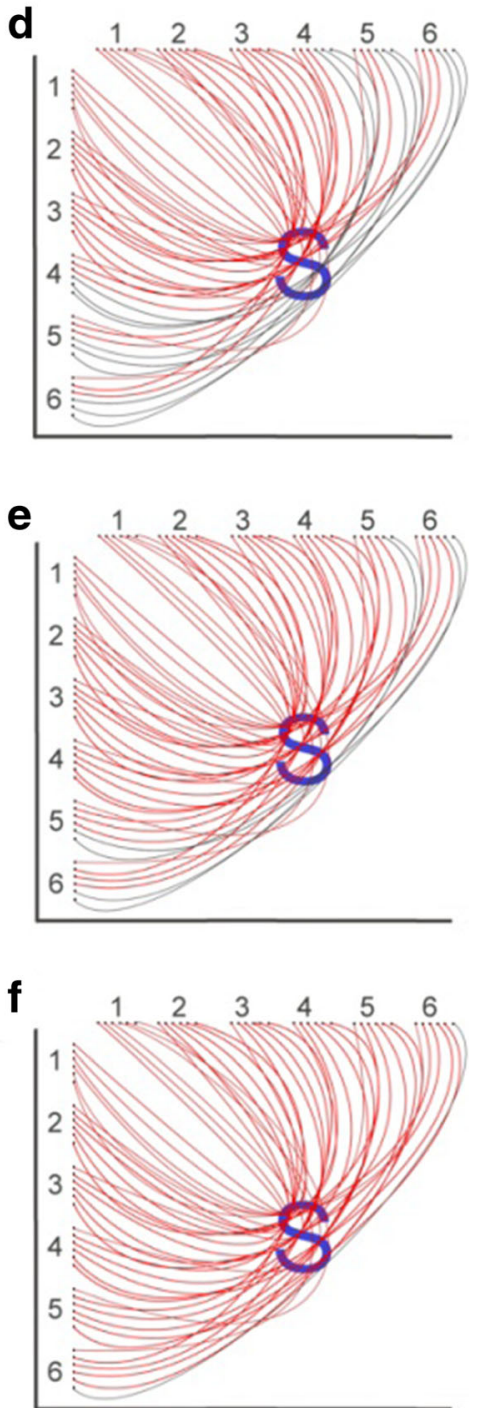

the first word $N^{\alpha+1}=36$ favorable relations exist and 0 are suppressed. B: Before naming the second word $(N-1)^{\alpha+1}=25$ favorable relations exist and $N^{\alpha+1}-(N-1)^{\alpha+1}=11$ are suppressed. C: Before naming the third word $(N-2)^{\alpha+1}=16$ favorable relations exist and $N^{\alpha+1}-$ $(N-2)^{\alpha+1}=20$ are suppressed. D: Before naming the fourth word: $(N-3)^{\alpha+1}=9$ favorable relations exist and $N^{\alpha+1}-(N-3)^{\alpha+1}=17$ are suppressed. E: Before naming the fifth word: $(N-4)^{\alpha+1}=4$ favorable relations exist and $N^{\alpha+1}-(N-4)^{\alpha+1}=32$ are suppressed. F: Before naming the sixth word: $(N-5)^{\alpha+1}=1$ favorable relations exist and $N^{\alpha+1}-(N-5)^{\alpha+1}=35$ are suppressed

items should trigger their underlying semantic concept (Muller et al., 2010; Vonberg et al., 2014), cross-domain coactivation can be expected by the time a word is articulated.

According to our model, Rule II (i.e., prohibition of repetitions) would therefore provoke suppression of the entire conglomerate of associations. Larger $\alpha$ values, indicating a higher degree of relatedness, would therefore occasion a larger decline of IRTs in the course of VF. Furthermore, due to Rule I (i.e., only words belonging to the task criterion), coactivation 
of related items may in large part hinder a rapid but accurate VF performance by facilitating access to multiple associations incompatible with the task criterion. Accordingly, the effort to constantly activate new and unrelated lexical entries (Robinson, Shallice, Bozzali, \& Cipolotti, 2012), while suppressing automatically coactivated items (Binder et al., 2009; Hickok \& Poeppel, 2007; Jackson et al., 2015), should bring about an increasing prefrontal executive involvement.

However, empirical data typically contain production spurts indicative of additional facilitatory effects that can be ascribed to coactivation (Foygel \& Dell, 2000; Raaijmakers \& Shiffrin, 1980; Rohrer, 1996, 2002; Rohrer \& Wixted, 1994; Shiffrin, 1970; Unsworth, Brewer, \& Spillers, 2013; Unsworth \& Engle, 2007; Vonberg et al., 2014). In this sense, the concomitant suppressing of potentially advantageous associations in the course of VF may also lead to an impoverishment of "access ways" to further suitable words leading to a greater retrieval effort (i.e., longer IRTs). As an example, the word tiger can be approached from the concept "cat," its association to "lion," or "Bengal," the features "stripe" or "fierce," or even from a semantically distant, but phonemically related word such as "Riga" as in the limerick "There was a young lady of Riga." Suppressing "lion" would still leave several other access ways open, yet they would narrow in as the course of VF continued.

Regarding this last consideration, however, the intended smoothing of local deviations by curve fitting approaches prevents the assessment of production spurts as possible indicators of facilitated lexical access. However, gaining access to this "micro structure" (D. J. Meyer et al., 2012. p. 213) may be achieved in future studies with cluster analysis using the FBF.

\section{Discussion}

Word production is viewed as a function of complex language-network interactions. Within this context, the here provided analysis of word production dynamics during VF tasks is intended to supplement the growing body of scientific knowledge regarding the processes involved. The analysis encompasses the design of a curve fitting function for VF dynamics and a subsequent model designed to yield further insights into underlying word retrieval processes. Despite the natural limitations of transferability to the assumed language network, the application of the thus obtained function to empirical data proved highly valuable.

Our approach is unique in that it is based on the two classical Bousfieldian functions, which resolves several uncertainties regarding their derivation and overcomes their apparent dichotomy. This is achieved by merging them into a single fused Bousfieldian function (FBF) family, thus qualifying them as special cases. The family parameter $\alpha$ was identified as the factor that modulates the shape of the corresponding curves. Furthermore, the initial rate $r$ was constructed from former Bousfieldian parameters, while $c$ was maintained as the asymptote. In so doing, a formerly unrevealed logarithmic time course became apparent as the limiting function of the family.

It is worth noting here that Bousfield and coworkers had previously suggested extending their hyperbolic approach (Bousfield et al., 1954). However, this endeavor might have been hindered before for primarily two reasons: (1),. The double denotation of the parameter $m$ in the expression of both functions precluded their unification. (2) The historically developed allocation of the exponential function to VF and of the hyperbolic function to (reinforced) verbal recall tasks most likely broadened this gap. Since both formulae often show strongly overlapping variances in their fittings, no occasion was provided to challenge the successful parallel use of both Bousfieldian formulae (cf. Gruenewald \& Lockhead, 1980). This perspective culminated in the dictum that "one cannot decide whether the data are better fit by an exponential curve or by a hyperbolic curve" (Herrmann \& Pearle, 1981, p. 148) The FBF offers an answer for this question of choice. Furthermore, it addresses the "efficiency problem."

Regarding the modeling of the FBF, our approach was again based on a method ascribed to Bousfield (i.e., the sampling-with-replacement model). The latter takes into account processes of lexical search, retrieval, and suppression (Bousfield \& Cohen, 1953b; Bousfield \& Sedgewick, 1944; Raaijmakers \& Shiffrin, 1980; Roediger \& Tulving, 1979; Rohrer, 1996, 2002; Rohrer \& Wixted, 1994; Shiffrin, 1970; Unsworth et al., 2013; Unsworth \& Engle, 2007). Expanding it for the FBF suggested the mental processing of highly dimensional lexical conglomerations rather than that of single items, with $\alpha$ representing the degree of their relatedness. The here formulated tuple activation and suppression (TAS) model proposes that the corresponding time course results from the suppression of connections to related items in addition to the suppression of already produced words. That is to say, taskspecific demands may induce increasing cognitive effort, which suppresses automatic network processes. This view emphasizes not only the constant effects of an unconscious interplay between countless word features represented across widespread cortical areas (Binder et al., 2009; Hickok \& Poeppel, 2007; Jackson et al., 2015) but also the large impact of task structure on processing strategies. In this context, it is of apparent interest that the initial rate $r$ was higher in semantic than in phonemic VF tasks. Since $r$ was defined as the reciprocal of the elementary process duration, i.e. the time needed to retrieve a correct word, corresponding process durations were about 1.54 seconds in semantic and 2.08 seconds in phonemic tasks. This general protraction of word production relative to the average speed of spoken German language (i.e., 2.2 words per second; Gebhard, 2012, p. 111) can be considered a result of specific VF demands. The particular 
prolongation in relation to phonemic tasks is in line with the view that phonemic tasks depend more strongly on frontal executive functions (for a review, see J. D. Henry \& Crawford, 2004a) in order to restrain inadequate semantic coactivation (Robinson et al., 2012). With respect to the growing body of knowledge regarding process sequencing within the language network (Costa et al., 2009; Dell, 1986; Levelt, 1999; Walker \& Hickok, 2015), phonemic VF may be visualized as a partial "inversion" of the usual processing direction.

Furthermore, values of $r$ were lower in the elder study group compared to the younger study group, indicating longer process durations. It is worth noting that the total number of produced words $N$ was not found to be lower in either the elder study group or under phonemic task conditions, suggesting that the final lexical output is largely independent of the elementary process duration.

Although TAS does not differentiate between different degrees of connection strength, it is reminiscent of two extensions of the sampling-with-replacement model, which address the influence of word connection on word production patterns, that is, the search of associative memory model (SAM; Shiffrin, 1970, Raaijmakers \& Shiffrin, 1980) and the two-stage model of free recall (Rohrer, 1996, 2002; Rohrer \& Wixted, 1994; cf. Unsworth et al., 2013; Unsworth \& Engle, 2007). However, although these models offer a plausible illustration of possible underlying mechanisms, they had not been intended as solutions for the dichotomy of the classical formulae.

Their fusion brought to light a previously unidentified logarithmic shape found in more than $70 \%$ of our data sets. Here the parameters $c$ and $\alpha$ approached infinity proportionally to one other. The divergence towards infinity underlines the character of the model in terms of both the capacity $c$ (Bousfield \& Sedgewick, 1944, p. 161; D. J. Meyer et al., 2012, p. 215) as well as the complexity represented by $\alpha$. Hence, the increase in $c$, which was originally understood as storage capacity, and $\alpha$, which can be interpreted as the degree of connectedness of the storage, are, coupled, and $c$ is renormalized by $\alpha$.

According to Prigogine, maximum self-similarity is preferable due to maximum efficiency of energy conversion during an efflux period (Glansdorff \& Prigogine, 1971; Prigogine, 1961). Here, evidence of this characteristic was provided by the highest degree of autocorrelation in the event of logarithmic time courses relative to the other curves (see Appendix 3). In this sense, the predominance of logarithmic time courses may indicate a pursuit of energy efficiency during VF tasks.

The assessment of autocorrelation constitutes a link between the empirical data, respectively, their curves, and the modeling. It therefore has a control function for the model's coherence. With respect to TAS, this becomes evident by the fact that the modeling is characterized qualitatively only by the parameter $\alpha$ that expresses relatedness and tuple structure, whereas the parameters $r$ and $c$ only cause quantitative modification. The same holds true for the autocorrelation of the curves which are exclusively parameterized by $\alpha$ but not by $r$ or $c$. Accordingly, the TAS modeling is in coherence with the network structure suggested by the curves.

A logarithmic function has also been applied by Luo and coworkers (Luo et al., 2010). However, this intuitively chosen approach has a singularity at $t=0$ and therefore does not represent the optimal procedure.

The numerical validity of the model's extension presented here was estimated by the Bayesian information criterion (BIC), with the standard deviation of curve fittings serving as the target value. The smallest deviations were almost always found in relation to graphs from the FBF family that were distinct from the two classical functions, yielding improvements of on average $20 \%$. This increase in accuracy outweighed the effort caused by the enlargement of the parameter set, as indicated by better BIC values relative to either the exponential ( $\sim 161 \%$ improvement) or the hyperbolic ( $\sim 19 \%$ improvement) functions.

Different attempts have been made previously to increase the quality of fitting functions by escalating the models' complexity (Gruenewald \& Lockhead, 1980; Wixted \& Rohrer, 1994). Even a sigmoidal approximation was discussed in terms of a Weibull (D. J. Meyer et al., 2012) or a Lévy distribution (Rhodes \& Turvey, 2007). However, since all of the cumulative curves examined in the current study had a negative curvature throughout, the latter approaches do not appear suitable here. In contrast to these works that have adopted new mathematical approaches, our formula is derived from already existing classical functions. Accordingly, the here presented method reduces the model's complexity both numerically - as expressed by the BIC (Schwarz, 1978; cf. Kahana et al., 2007; Myung \& Pitt, 1997) — and conceptually as demanded by Occam's law (Occam's Razor, 2015).

Interestingly, reanalysis of the data obtained by Bousfield and coworkers during verbal recall tasks with increasing degrees of reinforcement (Bousfield et al., 1954) by the FBF also indicated an improvement of the fitting results by roughly $50 \%$ and various values of $\alpha$ reaching from .5 to 8 (see Appendix 2). This would make the FBF also appear to be suitable for verbal recall tasks and demonstrates that, in addition to hyperbolic time courses, it is also possible to find courses in between the hyperbolic and the exponential function as well as courses with markedly larger values of $\alpha$. Furthermore, our reanalysis suggests an inverse tendency between the degree of reinforcement $R$ and $\alpha$, which indicates the clearly highest value of $\alpha$ in relation to just one presentation and is reminiscent of values found in VF tasks. This large value of $\alpha$ might indicate a naturally high number of coactivations if uninfluenced by reinforcement. Rehearsal of the list of words markedly lowered $\alpha$. According to our model, this could arise from overlearning effects resulting in a constriction of coactivated items.

Future studies applying the FBF in order to investigate word production dynamics as a function of lexical connectedness may shed further light on the ideas conceptualized here. 


\section{Conclusion}

The current study formulates a more advanced fitting function for word production dynamics during verbal fluency tasks. This fused Bousfieldian function (FBF) incorporates the two classical Bousfieldian functions that have been viewed as dichotomous for more than 60 years. In this way, the FBF yields a family of functions containing a previously unidentified logarithmic time course. Applying this to empirical data not only revealed a predominance of the logarithmic course, but also an improvement of fit by $20 \%$ while fulfilling the Bayesian information criterion. This served as the basis for developing a model that suggests, in particular, the existence of a connective structure between lexical storage and the function's shape parameter representing the degree of connectedness. Since it differentiates coactivation processes from increasing suppression effort, the FBF may serve to quantify production dynamics during VF and similar tasks, which presumes an interplay between automatic network effects and frontal executive functions.

Author note Supported by the German Research Foundation (K1-1276/ 5 in Clinical Research Group 247; Kl-1276/4-2).

\section{Compliance with ethical standards}

Conflict of interest The authors have no conflicts of interest.

\section{Appendix 1}

Let $T i, i=1, . ., I$ be the points of time where the cumulative number of words produced reaches the value $i=1, . ., I$. The parameters $c, r$, and $\alpha$ of the approximation $n(t)=n(c, r$, $\alpha, t)=C \cdot\left[1-(1+\alpha \cdot r \cdot t / c)^{-1 / \alpha}\right]$ now have to be determined in a manner so that the squared sum of deviations $Q=(c, r, \alpha)=\sum_{i=1}^{I}\left(n\left(T_{i}\right)-i\right)^{2}$ is minimalized. The disappearance of the first derivations $Q_{c}=\partial Q(c, r$, $\alpha) / \partial r, Q_{r}=\partial Q(c, r, \alpha) / \partial r$ and $Q_{\alpha}=\partial Q(c, r, \alpha) / \partial \alpha$ is necessary to obtain the minimum. Their Taylor series up to the first order are:

$Q_{c}(c \quad, r, \alpha)=Q_{c c} \cdot\left(c-c_{0}\right)+Q_{c r} \cdot\left(r-r_{0}\right)+Q_{c \alpha} \cdot\left(\alpha-\alpha_{0}\right)$, $Q_{r}(c, r, \alpha)=Q_{r c} \cdot\left(c-c_{0}\right)+Q \quad{ }_{r r} \cdot\left(r-r_{0}\right)+Q_{r \alpha} \cdot\left(\alpha-\alpha_{0}\right), Q_{\alpha}$ $(c, r, \alpha)=Q_{\alpha c} \cdot\left(c-c_{0}\right)+Q_{\alpha r} \cdot\left(r-r_{0}\right)+Q_{\alpha \alpha} \cdot\left(\alpha-\alpha_{0}\right)$ where $Q_{c c}, Q_{c r}$ etc. are the second partial derivations of $Q$, which on whole constitute the $3 \times 3$ Hessian Matrix $H$. By this means, Newton's method can be applied based on analytical expressions, free from further approximations.

It is true that $Q_{c}(c, r, \alpha)=\sum_{i=1}^{I} 2\left(n\left(T_{i}\right)-i\right) \cdot n_{c}\left(T_{i}\right)$ etc. and etc. and $Q_{c}(c, r, \alpha)=2 \sum_{i=1}^{I}\left(n_{c}(T)^{2}-\left(n\left(T_{i}\right)-i\right) \cdot n_{c c}\left(T_{i}\right)\right)$ etc., as well as $Q_{c r}(c, r, \alpha)=2 \sum_{i=1}^{I}\left(n_{r}\left(T_{i}\right) \cdot n\left(T_{i}\right)+\left(n\left(T_{i}\right)-i\right) \cdot n_{c r}\right.$ $\left.\left(T_{i}\right)\right)$ etc. Therefore all partial derivations of the first and second order of $n(c, r, \alpha, t)$ are needed. Using the abbreviations $A=r \alpha t+c, B=c / A$, and $C B^{1 / \alpha}$ it is true that:

$$
\begin{array}{ll}
n=c(1-C) ; & n_{c}=1-(A+r t) \cdot C / A ; \quad n_{r}=c t \cdot C / A ; \\
& n_{\alpha}=c C(r \alpha t+A \ln B) /\left(\alpha^{2} A\right)
\end{array}
$$

$n_{c c}=-C r^{2} t^{2} \cdot(\alpha+1) /\left(c A^{2}\right) ; \quad n_{r r}=-C r t^{2} \cdot(\alpha+1) / A^{2}$

$n_{\alpha \alpha}=-c C \cdot\left(\alpha^{2} A\right)^{-2}$

$\cdot\left[2 \alpha A(r t+A) \cdot \ln B+(A \cdot \ln B)^{2}+\alpha^{2} r t \cdot(r t \cdot(1+3 \alpha)+2 c)\right]$

$n_{c r}=C r t^{2} \cdot(\alpha+1) / A^{2}$

$n_{r \alpha}=-c t C \cdot(A \cdot \operatorname{in} B+\alpha r t \cdot(1+\alpha)) /(\alpha A)^{2}$;

$n_{c \alpha}=C(\alpha A)^{-2} \cdot[A \cdot(A+r t) \cdot \ln B+\alpha r t \cdot(r t \cdot(2 \alpha+1)=c]$.

Due to the analytic availability of the Hessian Matrix, it can be decided for each point of the parameter space whether $H$ is positive-definite or not. For a positivedefinite origin the iteration path remains within the positivedefinite area and leads to the requested minimum of $Q$.

\section{Appendix 2}

\section{Application of function (3a) to verbal recall data obtained by bousfield and Co-workers}

We used the power function (3a) to re-analyze the data from verbal recall tasks with varying degrees of reinforcement performed by Bousfield and co-workers who had been able to demonstrate a strong correlation between the degree of reinforcement (labeled here as $R$ ) and the asymptote $c$. In their study, five groups of participants $(n=49,52,46,49,47)$ were presented with a study list containing sixty words either once (i.e. no reinforcement; group I), twice (group II), three times (group III), four times (group IV), or five times (group V) and were asked to write down as many words as they could recall within ten minutes. The authors determined the cumulative number of words for each two-minute section and provided mean group values (Bousfield et al., 1954). We compared the results yielded by the application of function (3a) to those resulting from the use of the two classical functions, i.e. the exponential function $n(t)=c \cdot\left(1-e^{-r \cdot t / c}\right)$ and the hyperbolic function $n(t)=$ cr $t /(c+r t)$. As detailed above, data fitting was achieved using a $2 \times 2$ Hessian Matrix for the two classical functions (1a) and (2a), and a $3 \times 3$ Hessian Matrix for the power function (3a).

All curves were fitted successfully. Due to the relatively small number of available data sets, we 
refrained from analyzing the comparative data statistically and instead present the results descriptively (see Table 1):

Approximation performed by the power function yielded the lowest standard deviations $(\sigma)$ in all five cases. In line with the finding by Bousfield and coworkers, the fit of the hyperbolic function was superior to that of the exponential function. Values of the asymptote $c$ were primarily highest when applying the power function and lowest in relation to the exponential function. The observation made by Bousfield and coworkers that the rate of word production increases with more frequent presentations of the study list was indicated in our re-analysis by a tendency towards an increase in the newly introduced initial rate $r$ as a function of $R$ in relation to the two classical Bousfieldian functions. Their comparison shows that all $r$-values are approximately $40 \%$ larger if applying (2a) rather than (1a). This verifies our initial analysis, indicating that in order to produce an actual approximation, the hyperbolic course can compensate for its naturally flatter curve only with a comparably larger initial rate. Further evidence is provided by the fact that approximations performed by the power function do not show this tendency. Instead, both the data values and the optical impression indicated a globally homogenous initial $r$, independent from $R$. Having said this, the further time course was clearly flatter the more often the viewer was presented with the list. Altogether, the data stemming from the power function corroborate our initial criticism concerning the initial rate as an argument in favor of the hyperbolic function.

Regarding the approximation by the power function (see also Fig. 9), values of $\alpha$ differed markedly with a tendency to decrease as $R$ increased. In the case of just

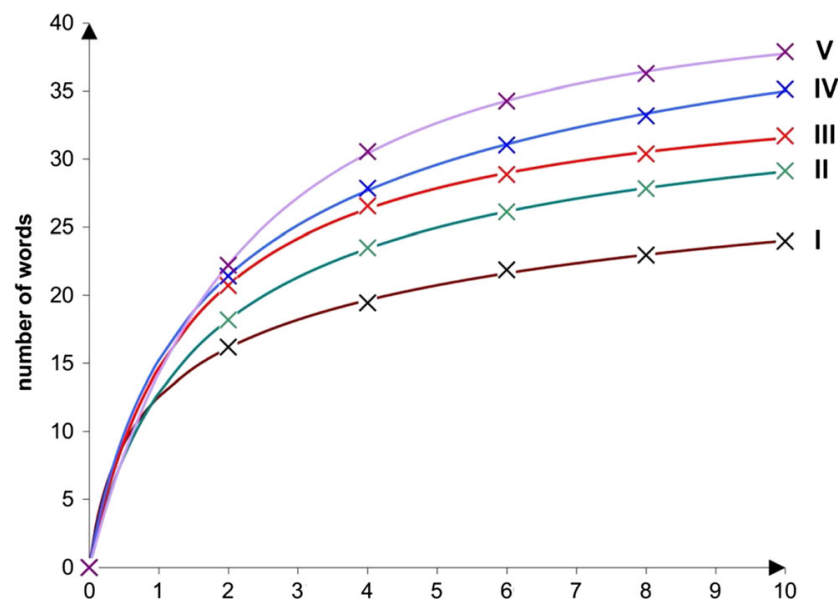

Fig. 9 The data obtained by Bousfield and co-workers from verbal recall tasks after presenting the study list one, two, three, four, or five times to five groups of students (I-V) (Bousfield et al., 1954) were re-analyzed using the power function (3a). The approximation delivered an improved fit and indicated distinct curve shapes due to a variable exponent

one presentation, i.e. no reinforcement (group I), a comparably large $\alpha$ value of approximately eight was found along with the highest asymptote and a long halflife, so that its approach would only become visible far beyond the ten minute interval. With these characteristics, the case of one presentation indicated a trend towards a logarithmic shape, where the asymptote-lying in infinity-is never reached. The value of approximately one (1) in group III fit well with a hyperbolic course. In groups II and IV, $\alpha$ values of approximately two (2) and three (3), respectively, indicated more protracted time courses, while a value of 0.5 in group $\mathrm{V}$ yielded a time course in between the exponential and the hyperbolic.

Table 1 Results from a re-analysis of the data provided by Bousfield and co-workers by the exponential, hyperbolic, and power functions are provided

\begin{tabular}{|c|c|c|c|c|c|c|c|c|c|c|c|c|c|c|c|}
\hline \multirow[t]{2}{*}{ Group i. e. $\mathrm{R}$} & \multirow[t]{2}{*}{ Words mean $( \pm \mathrm{SD})$} & \multicolumn{4}{|c|}{ Exponential Function } & \multicolumn{4}{|c|}{ Hyperbolic Function } & \multicolumn{6}{|c|}{ Power Function } \\
\hline & & $\sigma$ & $c$ & $r$ & $t H$ & $\sigma$ & $c$ & $r$ & $t H$ & $\sigma$ & $c$ & $r$ & $t H$ & $k$ & $\alpha$ \\
\hline$I$ & $23.96( \pm 6.69)$ & .35 & 23.24 & 12.52 & 1.29 & .14 & 27.04 & 19.14 & 1.41 & .07 & 61.09 & 40.33 & 47.54 & .13 & 7.97 \\
\hline II & $29.12( \pm 3.65)$ & .28 & 28.54 & 13.42 & 1.47 & .06 & 34.05 & 19.21 & 1.77 & .01 & 39.70 & 22.76 & 2.47 & .05 & 1.86 \\
\hline III & $31.7( \pm 3.65)$ & .26 & 30.94 & 16.20 & 1.32 & .06 & 36.22 & 24.27 & 1.49 & .06 & 36.93 & 25.06 & 1.54 & .03 & 1.11 \\
\hline IV & $35.12( \pm 3.65)$ & .34 & 34.32 & 15.45 & 1.54 & .11 & 41.28 & 21.73 & 1.90 & .04 & 57.08 & 28.65 & 4.41 & .05 & 2.89 \\
\hline V & $37.87( \pm 3.65)$ & .18 & 37.71 & 16.12 & 1.62 & .09 & 45.90 & 22.06 & 2.08 & .04 & 41.48 & 19.50 & 1.77 & .01 & 0.51 \\
\hline
\end{tabular}

The original data sets stemming from verbal recall tasks with varying degrees of reinforcement $(R)$ had been obtained from five groups (I-V), which had been presented with the study list of sixty words one, two, three, four, or five times respectively. The cumulative number of words was provided as group mean values (and standard deviations). Slight differences between the here presented values of $c$ and $t_{H}$ compared to those given in the original study result from different approximation methods (in this case with a Hessian Matrix, in the original study "a method of averages" (Bousfield et al., 1954; page 115)

$\sigma:$ accuracy of fit; $c$ : asymptote (in words); $r$ : initial rate (in words/min); $t_{H}$ : half-life (in min); $\alpha$ : shape parameter; $k: c / \alpha$ 


\section{Appendix 3}

\section{Calculation of autocorrelations of the density distributions}

Density functions $\rho(t)=n^{\prime}(t)$ were determined as derivation with respect to time of the cumulated exponential, hyperbolic, potential, and logarithmic time courses $n(t)$. Autocorrelations of the respective density functions were computed as a function of the rescaling factor $p$ using the formula $\gamma(p)=$ $T \rightarrow \infty \lim \frac{\delta(p, T)-\mu(T) \mu_{p}(T)}{\sigma(T) \sigma_{p}(T)}$, with $\mu(T)=\frac{1}{T} \int_{0}^{T} \rho(t) d t, \mu_{p}(T)=$ $\frac{1}{T} \int_{0}^{T} \rho(p t) d t, \delta(p, T)=\frac{1}{T} \int_{0}^{T} \rho(t) \cdot \rho(p t) d t, q(T)=\frac{1}{T} \int_{0}^{T} \rho(t)^{2} \cdot \rho$ $(t)^{2} d t, \sigma(T)=\sqrt{q}(T)-\mu(T)^{2}, q_{p}(T)=\frac{1}{T} \int_{0}^{T} \rho(p t)^{2} d t$, and $\sigma_{\mathrm{p}}(T)=\sqrt{q_{p}(T)}-\mu_{p}(T)^{2}$. The results are provided in Table 2. Corresponding graphs are illustrated in Fig. 7.

Table 2 The course of autocorrelation $(\gamma)$ was determined as a function of the rescaling factor $(p)$ for the exponential (EXP), hyperbolic (HYP), power, and logarhithmic (LOG) function

\begin{tabular}{|c|c|c|c|c|c|}
\hline Function & $\alpha$ & $\tau$ & $n(t)$ & $\rho(t)$ & $\gamma(p)$ \\
\hline EXP & 0 & $\mathrm{c} / \mathrm{r}$ & $c\left(1-e^{-1 / \tau}\right)$ & $\frac{c}{\tau} \cdot e^{-t / \tau}$ & $\frac{2 \sqrt{p}}{p+1}$ \\
\hline HYP & 1 & $\frac{c}{\alpha r}$ & $c\left(1-\frac{\tau}{t+\tau}\right)$ & $\frac{c}{\tau} \cdot\left(\frac{\tau}{t+\tau}\right)^{2}$ & $\frac{3\left(p^{2}-1-2 p \ln p\right) \sqrt{p}}{(p-1)^{3}}$ \\
\hline Power & $a r b$. & $\frac{c}{\alpha r}$ & $c\left(1-\left(\frac{\tau}{t+\tau}\right)\right.$ & $\frac{c}{\tau \alpha} \cdot\left(\frac{\tau}{t+\tau}\right)^{\frac{1}{\alpha}+1}$ & $\begin{array}{l}\text { Determined } \\
\text { numerically }\end{array}$ \\
\hline LOG & $\rightarrow \infty$ & $\frac{k}{r}$ & $-k \cdot \ln \frac{\tau}{t+\tau}$ & $\frac{k}{\tau} \cdot \frac{\tau}{t+\tau}$ & $\frac{\sqrt{p \cdot \ln p}}{p-1}$ \\
\hline
\end{tabular}

In case of the power function, the $\alpha$ value can be chosen arbitrarily (arb.), and here the degree of autocorrelation was determined numerically for each $p$. For the other three functions, formulae for autocorrelation were developed (as given in the final row). Remark: $\gamma(p)$ is independent of $r$ and $c$, respectively $r$ and $k$

$\alpha$ : shape parameter; $\tau$ : time constant $(\mathrm{s}) ; n(t)$ : cumulative number of produced words as function of time $(t) ; \rho(t)$ : density distribution as function of time; $c$ : asymptote; $k$ : coupling constant $(\mathrm{c} / \alpha)$

\section{References}

Albert, D. (1968). Freies Reproduzieren von Wortreihen als stochastische Entleerung eines Speichers. Zeitschrift für experimentelle und angewandte Psychologie, 15, 564-581.

Albert, R., \& Barabasi, A. L. (2002). Statistical mechanics of complex networks. Reviews of Modern Physics, 74(1), 47-97. doi:10.1103/ RevModPhys.74.47

Apfelbaum, K. S., Blumstein, S. E., \& McMurray, B. (2011). Semantic priming is affected by real-time phonological competition: Evidence for continuous cascading systems. Psychonomic Bulletin and Review, 18(1), 141-149. doi:10.3758/s13423-010-0039-8

Aschenbrenner, A., Tucha, O., \& Lange, K. (2000). RWT Regensburger Wortflüssigkeits-Test. Handanweisung. Göttingen: Hogrefe Verlag.

Baldo, J. V., Shimamura, A. P., Delis, D. C., Kramer, J., \& Kaplan, E. (2001). Verbal and design fluency in patients with frontal lobe lesions. Journal of International Neuropsychological Society, 7(5), 586-596.

Bauml, K. H., Zellner, M., \& Vilimek, R. (2005). When remembering causes forgetting: Retrieval-induced forgetting as recovery failure. Journal of Experimental Psychology: Learning Memory and Cognition, 31(6), 1221-1234. doi:10.1037/0278-7393.31. 6.1221

Binder, J. R., Desai, R. H., Graves, W. W., \& Conant, L. L. (2009). Where is the semantic system? A critical review and meta-analysis of 120 functional neuroimaging studies. Cerebral Cortex, 19(12), 27672796. doi:10.1093/cercor/bhp055

Bousfield, W. A., \& Cohen, B. H. (1953a). The effects of reinforcement on the occurrence of clustering in the recall of randomly arranged associates. The Journal of Psychology, 36(1), 67-81. doi:10.1080/ 00223980.1953 .9712878

Bousfield, W. A., \& Cohen, B. H. (1953b). The occurrence of clustering in the recall of randomly arranged associates. The Journal of Psychology, 36, 67-81.

Bousfield, W. A., \& Sedgewick, C. H. W. (1944). An analysis of sequences of restricted associative responses. The Journal of General Psychology, 30(2), 149-165.

Bousfield, W. A., Sedgewick, C. H., \& Cohen, B. H. (1954). Certain temporal characteristics of the recall of verbal associates. American Journal of Psychology, 67(1), 111-118.

Braun, M., Jacobs, A. M., Richlan, F., Hawelka, S., Hutzler, F., \& Kronbichler, M. (2015). Many neighbors are not silent. fMRI evidence for global lexical activity in visual word recognition. Frontiers in Human Neuroscience, 9(423). doi:10.3389/fnhum. 2015.00423

Caramazza, A. (1997). How many levels of processing are there in lexical access? Cognitive Neuropsychology, 14(1), 177-208. doi:10.1080/ 026432997381664

Collins, A. M., \& Loftus, E. F. (1975). A apreading-activation theory of semantic processing. Psychological Review, 82(6), 407-428.

Costa, A., Strijkers, K., Martin, C., \& Thierry, G. (2009). The time course of word retrieval revealed by event-related brain potentials during overt speech. Proceedings of the National Acadamy of Sciences of the United Sates of America, 106(50), 21442-21446. doi:10.1073/ pnas.0908921106

Crosson, B. (2013). Thalamic mechanisms in language: A reconsideration based on recent findings and concepts. Brain and Language, 126(1), 73-88. doi:10.1016/j.bandl.2012.06.011

Crosson, B., Benjamin, M., \& Levy, I. (2007). Role of the basal ganglia in language: Supporting cast. In J. H. M. A. Kraut (Ed.), Neural basis of semantic memory (pp. 219-233). New York: Cambridge University Press.

Dell, G. S. (1986). A spreading-activation theory of retrieval in sentence production. Psychological Review, 93(3), 283-321. doi:10.1037// 0033-295x.93.3.283

Duff, K., Schoenberg, M. R., Scott, J. G., \& Adams, R. L. (2005). The relationship between executive functioning and verbal and visual learning and memory. Archives of Clinical Neuropsychology, 20(1), 111-122. doi:10.1016/j.acn.2004.03.003

Faul, F., Erdfelder, E., Lang, A. G., \& Buchner, A. (2007). G*Power 3: A flexible statistical power analysis program for the social, behavioral, and biomedical sciences. Behavior Research Methods, 39(2), 175191. doi:10.3758/Bf03193146

Fitzgerald, J. M. (1983). A developmental-study of recall from natural categories. Developmental Psychology, 19(1), 9-14. doi:10.1037// 0012-1649.19.1.9 
Foygel, D., \& Dell, G. S. (2000). Models of impaired lexical access in speech production. Journal of Memory and Language, 43(2), 182216. doi:10.1006/jmla.2000.2716

Gebhard, C. (2012). Sprechtempo im sprachvergleich: Eine intersuchung phonologischer und kultureller aspekte anhand von nachrichtensendungen [Speech tempo in contrastive linguistics: An analysis of phonological and cultural aspects by means of news broadcast] (Doctoral thesis, Humboldt-Universität zu Berlin, Berlin).

Glansdorff, P. G., \& Prigogine, I. (1971). Thermodynamic theory of structure, stability and fluctuations. New York: Wiley.

Graesser, A., \& Mandler, G. (1978). Limited processing capacity constrains the storage of unrelated sets of words and retrieval from natural categories. Journal of Experimental Psychology: Human Learning \& Memory, 4(1), 86-100. doi:10.1037/0278-7393.4.1.86

Gruenewald, P. J., \& Lockhead, G. R. (1980). The free-recall of category examples. Journal of Experimental Psychology: Human Learning and Memory, 6(3), 225-240. doi:10.1037//0278-7393.6.3.225

Hart, J., Jr., Maguire, M. J., Motes, M., Mudar, R. A., Chiang, H. S., Womack, K. B., \& Kraut, M. A. (2013). Semantic memory retrieval circuit: Role of pre-SMA, caudate, and thalamus. Brain and Language, 126(1), 89-98. doi:10.1016/j.band1.2012.08.002

Henry, J. D., \& Crawford, J. R. (2004a). A meta-analytic review of verbal fluency performance following focal cortical lesions. Neuropsychology, 18(2), 284295. doi:10.1037/0894-4105.18.2.284

Henry, J. D., \& Crawford, J. R. (2004b). Verbal fluency deficits in Parkinson's disease: A meta-analysis. Journal of the International Neuropsychological Society, 10(4), 608-622. doi:10.1017/ S1355617704104141

Henry, J., \& Crawford, J. R. (2005). A meta-analytic review of verbal fluency deficits in depression. Journal of Clinical and Experimental Neuropsychology, 27(1), 78-101. doi:10.1080/138033990513654

Herrmann, D. J., \& Chaffin, R. J. S. (1976). Number of available associations and rate of association for categories in semantic memory. The Journal of General Psychology, 95(2), 227-231.

Herrmann, D. J., \& Murray, D. (1979). The role of category size in continuous recall from semantic memory. The Journal of General Psychology, 101(2), 205-218. doi:10.1080/00221309.1979. 9920075

Herrmann, D. J., \& Pearle, P. M. (1981). The proper role of clusters in mathematical-models of continuous recall. Journal of Mathematical Psychology, 24(2), 139-162. doi:10.1016/0022-2496(81)90040-7

Hickok, G. (2012). Computational neuroanatomy of speech production. Nature Reviews Neuroscience, 13(2), 135-145. doi:10.1038/ nrn2158

Hickok, G., \& Poeppel, D. (2007). The cortical organization of speech processing. Nature Reviews Neuroscience, 8(5), 393-402. doi:10. $1038 / \mathrm{nrn} 2113$

Indefrey, P. (2011). The spatial and temporal signatures of word production components: A critical update. Frontiers in Psychology, 2, 255. doi:10.3389/fpsyg.2011.00255

Indefrey, P., \& Levelt, W. J. M. (2000). The neural correlates of language production. In M. S. Gazzaniga (Ed.), The new cognitive neurosciences (2nd ed., pp. 845-865). Cambridge: MIT Press.

Indefrey, P., \& Levelt, W. J. (2004). The spatial and temporal signatures of word production components. Cognition, 92(1/2), 101-144. doi:10. 1016/j.cognition.2002.06.001

Indow, T., \& Togano, K. (1970). On retrieving sequence from long-term memory. Psychological Review, 77(4), 317-331. doi:10.1037/ H0029395

Jackson, R. L., Hoffman, P., Pobric, G., \& Lambon Ralph, M. A. (2015). The nature and neural correlates of semantic association versus conceptual similarity. Cerebral Cortex. doi:10.1093/cercor/bhv003

Johnson, D. M., Johnson, R. C., \& Mark, A. L. (1951). A mathematical analysis of verbal fluency. The Journal of General Psychology, 44(1), 121-128. doi:10.1080/00221309.1951.9711240
Kahana, N. I., Zhou, F., Geller, A. S., \& Sekuler, R. (2007). Lure similarity affects visual episodic recognition: Detailed tests of a noisy exemplar model. Memory \& Cognition, 35(6), 1222-1232.

Kaplan, I. T., Carvella, T., \& Metlay, W. (1969). Searching for words in letter sets of varying size. Journal of Experimental Psychology, 82(2), 377-380. doi:10.1037/h0028140

Levelt, W. J. (1999). Producing spoken language: A blueprint of the speaker. In I. C. B. P. Hagoort (Ed.), The neurocognition of language (pp. 83-122). Oxford: Oxford University Press.

Levelt, W. J., Roelofs, A., \& Meyer, A. S. (1999). A theory of lexical access in speech production. The Behavioral and Brain Sciences, 22(1), 1-38. discussion 38-75.

Lichtheim, L. (1885). Ueber Aphasie [On Aphasia]. Deutsches Archiv für Klinische Medizin, 36, 204268.

Luo, L., Luk, G., \& Bialystok, E. (2010). Effect of language proficiency and executive control on verbal fluency performance in bilinguals. Cognition, 114(1), 2941. doi:10.1016/j.cognition.2009.08.014

Mcclelland, J. L., \& Rumelhart, D. E. (1981). An interactive activation model of context effects in letter perception: 1 . An account of basic findings. Psychological Review, 88(5), 375-407. doi:10.1037/0033295x.88.5.375

McDermott, K. B., Petersen, S. E., Watson, J. M., \& Ojemann, J. G. (2003). A procedure for identifying regions preferentially activated by attention to semantic and phonological relations using functional magnetic resonance imaging. Neuropsychologia, 41(3), 293-303.

McGill, W. J. (1963). Stochastic latency mechanisms. In R. R. B. R. D. Luce \& E. Galanter (Eds.), Handbook of mathematical psychology (1st ed., pp. 309-360). New York: Wiley.

Meyer, D. J., Messer, J., Singh, T., Thomas, P. J., Woyczynski, W. A., Kaye, J., \& Lerner, A. J. (2012). Random local temporal structure of category fluency responses. Journal of Computational Neuroscience, 32(2), 213-231. doi:10.1007/s10827-011-0349-5

Meyer, D. E., \& Schvaneveldt, R. W. (1971). Facilitation in recognizing pairs of words: Evidence of a dependence between retrieval operations. Journal of Experimental Psychology, 90(2), 227-234. doi:10. 1037/H0031564

Muller, O., Dunabeitia, J. A., \& Carreiras, M. (2010). Orthographic and associative neighborhood density effects: What is shared, what is different? Psychophysiology, 47(3), 455-466. doi:10.1111/j.14698986.2009.00960.x

Myung, I. J., \& Pitt, M. A. (1997). Applying Occam's razor in modeling cognition: A Bayesian approach. Psychonomic Bulletin and Review, 4(1), 79-95. doi:10.3758/Bf03210778

Newman, M. E. J. (2005). Power laws, Pareto distributions and Zipf's law. Contemporary Physics, 46(5), 323-351. doi:10.1080/ 00107510500052444

Obeso, I., Casabona, E., Bringas, M. L., Alvarez, L., \& Jahanshahi, M. (2012). Semantic and phonemic verbal fluency in Parkinson's disease: Influence of clinical and demographic variables. Behavioural Neurology, 25(2), 111-118.

Occam's razor. (2015). In Encyclopcedia Britannica online. Retrieved from http://www.britannica.com/EBchecked/topic/424706/ Occams-razor

Pareto, V. (1897). Cours d'économie politique [Political Economy]. Switzerland: Lausanne.

Parks, R. W., Levine, D. S., Long, D. L., Crockett, D. J., Dalton, I. E., Weingartner, H., ... Becker, R. E. (1992). Parallel distributedprocessing and neuropsychology: A neural network model of Wisconsin card sorting and verbal fluency. Neuropsychology Review, 3(2), 213-233. doi:10.1007/Bf01108843

Pollio, H. R. (1964). Composition of associative clusters. Journal of Experimental Psychology, 67, 199-208.

Prigogine, I. (1961). Introduction to thermodynamics of irreversible processes. Springfield, Illinois, USA: Charles C. Thomas. 
Pulvermuller, F. (1999). Words in the brain's language. The Behavioral and Brain Sciences, 22(2), 253-279. discussion 280-336.

Raaijmakers, J. G. W., \& Shiffrin, R. M. (1980). SAM: A theory of probabilistic search of associative memory. In G. H. Bower (Ed.), The psychology of learning and motivation (Vol. 14, pp. 207-262). New York: Academic Press.

Rapp, B., \& Goldrick, M. (2000). Discreteness and interactivity in spoken word production. Psychological Review, 107(3), 460-499. doi:10. 1037/0033-295x.107.3.460

Rhodes, T., \& Turvey, M. T. (2007). Human memory retrieval as Levy foraging. Physica A: Statistical Mechanics and Its Applications, 385(1), 255-260. doi:10.1016/j.physa.2007.07.001

Robinson, G., Shallice, T., Bozzali, M., \& Cipolotti, L. (2012). The differing roles of the frontal cortex in fluency tests. Brain, 135(Pt. 7), 2202-2214. doi:10.1093/brain/aws142

Roediger, H. L., \& Tulving, E. (1979). Exclusion of learned material from recall as a post-retrieval operation. Journal of Verbal Learning and Verbal Behavior, 18(5), 601-615. doi:10.1016/S0022-5371(79) 90334-7

Roelofs, A. (1992). A spreading-activation theory of lemma retrieval in speaking. Cognition, 42(1/3), 107-142. doi:10.1016/0010-0277(92) 90041-f

Rohrer, D. (1996). On the relative and absolute strength of a memory trace. Memory and Cognittion, 24(2), 188-201. doi:10.3758/ Bf03200880

Rohrer, D. (2002). The breadth of memory search. Memory, 10(4), 291301. doi:10.1080/09658210143000407

Rohrer, D., \& Wixted, J. T. (1994). An analysis of latency and interresponse time in free-recall. Memory and Cognition, 22(5), 511-524. doi:10.3758/Bf03198390

Rohrer, D., Wixted, J. T., Salmon, D. P., \& Butters, N. (1995). Retrieval from semantic memory and its implications for Alzheimer's disease. Journal of Experimental Psychology: Learning, Memory, and Cognition, 21(5), 1127-1139.

Saur, D., Kreher, B. W., Schnell, S., Kummerer, D., Kellmeyer, P., Vry, M. S., ... Weiller, C. (2008). Ventral and dorsal pathways for language. Procedures of the National Academy of Sciences of the United States of America, 105(46), 18035-18040. doi:10.1073/ pnas.0805234105

Schuhmann, T., Schiller, N. O., Goebel, R., \& Sack, A. T. (2009). The temporal characteristics of functional activation in Broca's area during overt picture naming. Cortex, 45(9), 1111-1116. doi:10.1016/j. cortex.2008.10.013

Schwartz, M. F., Dell, G. S., Martin, N., Gahl, S., \& Sobel, P. (2006). A case-series test of the interactive two-step model of lexical access: Evidence from picture naming. Journal of Memory and Language, 54(2), 228-264. doi:10.1016/j.jml.2005.10.001

Schwarz, G. (1978). Estimating the dimension of a model. The Annals of Statistics, 6(2), 461-464.

Shannon, C. E. (1948). A mathematical theory of communication. The Bell System Technical Journal, 27(3, 4)379-423, 623-656.
Shiffrin, R. M. (1970). Memory search. In D. A. Norman (Ed.), Models of human memory (pp. 375-447). New York: Academic Press.

Shiffrin, R. M., \& Atkinson, R. C. (1969). Storage and retrieval processes in long-term memory. Psychological Review, 76(2), 179-193. doi: 10.1037/H0027277

Stein, J., Luppa, M., Brahler, E., Konig, H. H., \& Riedel-Heller, S. G. (2010). The assessment of changes in cognitive functioning: Reliable change indices for neuropsychological instruments in the elderly-A systematic review. Dementia and Geriatric Cogntive Disorders, 29(3), 275-286. doi:10.1159/000289779

Troyer, A. K., Moscovitch, M., \& Winocur, G. (1997). Clustering and switching as two components of verbal fluency: Evidence from younger and older healthy adults. Neuropsychology, 11(1), 138 146. doi:10.1037//0894-4105.11.1.138

Unsworth, N., Brewer, G. A., \& Spillers, G. J. (2013). Working memory capacity and retrieval from long-term memory: The role of controlled search. Memory and Cognition, 41(2), 242-254. doi:10. 3758/s13421-012-0261-x

Unsworth, N., \& Engle, R. W. (2007). The nature of individual differences in working memory capacity: Active maintenance in primary memory and controlled search from secondary memory. Psychological Review, 114(1), 104-132. doi:10.1037/0033-295x. 114.1.104

Vonberg, I., Ehlen, F., Fromm, O., \& Klostermann, F. (2014). The absoluteness of semantic processing: Lessons from the analysis of temporal clusters in phonemic verbal fluency. PLOS ONE, 9(12), e115846. doi:10.1371/journal.pone.0115846

Wahl, M., Marzinzik, F., Friederici, A. D., Hahne, A., Kupsch, A., Schneider, G. H., ... Klostermann, F. (2008). The human thalamus processes syntactic and semantic language violations. Neuron, 59(5), 695-707. doi:10.1016/j.neuron.2008.07.011

Walker, G. M., \& Hickok, G. (2015). Bridging computational approaches to speech production: The semantic-lexical-auditory-motor model (SLAM). Psychonomic Bulletin and Review. doi:10.3758/s13423015-0903-7

Wernicke, C. (1874). Der aphasische Symptomencomplex: Eine psychologische Studie auf anatomischer Basis. Breslau: Max Cohn \& Weigert.

Wilson, S. M., Isenberg, A. L., \& Hickok, G. (2009). Neural correlates of word production stages delineated by parametric modulation of psycholinguistic variables. Human Brain Mapping, 30(11), 3596-3608. doi: $10.1002 / \mathrm{hbm} .20782$

Wixted, J. T., \& Ebbesen, E. B. (1991). On the form of forgetting. Psychological Science, 2(6), 409-415. doi:10.1111/j.1467-9280. 1991.tb00175.x

Wixted, J. T., \& Rohrer, D. (1994). Analyzing the dynamics of free recall: An integrative review of the empirical literature. Psychonomic Bulletin and Review, 1(1), 89-106. doi:10.3758/BF03200763

Young, C. J. (2004). Contributions of metaknowledge to retrieval of natural categories in semantic memory. Journal of Experimental Psychology: Learning Memory and Cognition, 30(4), 909-916. doi:10.1037/0278-7393.30.4.909 